\title{
Patched regulates lipid homeostasis by controlling cellular cholesterol levels
}

Carla E. Cadena del Castillo ${ }^{1}$, J. Thomas Hannich ${ }^{2}$, Andres Kaech ${ }^{3}$, Hirohisa Chiyoda ${ }^{4}$, Masamitsu Fukuyama ${ }^{4}$, Nils J. Færgeman ${ }^{5}$, Howard Riezman ${ }^{2}$ and Anne Spang ${ }^{1}$

${ }^{1}$ Biozentrum, University of Basel, Switzerland ${ }^{2}$ Department of Biochemistry and NCCR Chemical Biology, University of Geneva, Switzerland, ${ }^{3}$ Center for Microscopy and Image Analysis, University of Zürich, Switzerland, ${ }^{4}$ Laboratory of Physiological Chemistry, Graduate School of Pharmaceutical Sciences, University of Tokyo, Tokyo, Japan, ${ }^{5}$ Department of Biochemistry and Molecular Biology, Villum Center for Bioanalytical Sciences, University of Southern Denmark, Odense, Denmark.

Corresponding Author:

Anne Spang

Biozentrum

University of Basel

Klingelbergstrasse 70

$\mathrm{CH}-4056$ Basel

Switzerland

Email: anne.spang@unibas.ch

Phone: +41612072380 


\section{Abstract (149 words)}

Hedgehog $(\mathrm{Hh})$ signaling is essential during development and in organ physiology. In the canonical pathway, Hh binding to Patched $(\mathrm{PTCH})$ relieves the inhibition of Smoothened (SMO). Yet, PTCH may also perform SMO-independent functions. While the PTCH homolog PTC-3 is essential in C. elegans, worms lack SMO, providing an excellent model to probe non-canonical PTCH function. Here, we show that PTC-3 is a cholesterol transporter. ptc-3(RNAi) leads to accumulation of intracellular cholesterol and defects in ER structure and lipid droplet formation. These phenotypes were accompanied by a reduction in acyl chain (FA) length and desaturation. ptc-3(RNAi)induced lethality, fat storage and ER morphology defects were rescued by reducing dietary cholesterol. We provide evidence that cholesterol accumulation modulates the function of nuclear hormone receptors such as of the PPAR $\alpha$ homolog NHR-49 and NHR-181, and affects FA composition. Our data uncover a novel role for PTCH in organelle structure maintenance and fat metabolism. 


\section{Introduction}

The Hedgehog $(\mathrm{Hh})$ signaling pathway is crucial during animal development and has also demonstrated roles independent of development stages. The Hh receptor PTCH is among the most mutated tumor suppressors ${ }^{1}$ and more specifically, PTCH1 mutations are the cause of the Gorlin Syndrome ${ }^{2}$. In the classical Hh signaling pathway, PTCH inhibits the plasma membrane G-protein coupled receptor (GPCR) smoothened (SMO). Upon Hh binding to PTCH, this inhibition is relieved, and SMO can activate a downstream signaling cascade. The mechanism by which PTCH inhibits SMO was enigmatic for a long time because PTCH represses SMO without direct contact ${ }^{3}$. PTCH1 was shown to be able to transport cholesterol ${ }^{4-6}$ which in turn will directly activate $\mathrm{SMO}^{7}$, a finding, which was supported by recent structural analyses ${ }^{1,8-10}$. The structures suggest that $\mathrm{Hh}$ inhibits PTCH transporter function and hence plasma membrane cholesterol levels could increase. Such an increase of cholesterol might be sensed through the sterol sensing domain in SMO and thereby activate the GPCR. As PTCH may mainly function as a cholesterol transporter, it might also affect other signaling pathways. In fact, in recent years SMO-independent PTCH signaling has been reported ${ }^{11-14}$. However, the mechanistic understanding of these non-canonical $\mathrm{Hh}$ signaling pathways remains largely unknown.

Caenorhabditis elegans expresses two PTCH homologs, PTC-1 and PTC-3, which are essential for development and survival ${ }^{15-17}$. While PTC-1 function appears to be mostly restricted to the germline, PTC-3 is expressed in somatic tissues ${ }^{18-20}$. No clear SMO homolog is encoded in the genome. In addition, some of the other downstream targets of the canonical Hh signaling pathway are also missing. In fact, it was proposed that SMO and those components were specifically lost during evolution in nematodes ${ }^{15,21-23}$. For example, SUFU is not conserved and the homolog of the transcription factor Gli, TRA-1, is involved in sex determination and gonad development in males and hermaphrodites ${ }^{24}$. Therefore, C. elegans provides an excellent model to study non-canonical, SMO-independent $\mathrm{Hh}$ signaling pathways, in particular in somatic tissues. To dissect SMO-independent PTCH functions, we concentrated on PTC-3, which is expressed in somatic tissues, in particular in the hypodermis, glia and gut ${ }^{20}$. We found that reduction of PTC-3 levels causes the accumulation of intracellular cholesterol and reduction in poly unsaturated fatty acids 
(PUFAs). Moreover, the endoplasmic reticulum lost most of its reticulate tubular form and developed elaborate sheet structures in the intestine. This effect in turn strongly impaired lipid droplet biogenesis, resulting in the inability of the animal to store fat. Reduction of dietary cholesterol rescued fat storage defects, the ER morphology defects, and improved development and survival in ptc-3(RNAi) animals. Cholesterol levels influence nuclear hormone receptor activity such as of the PPAR $\alpha$ homolog NHR-49, which is involved in the regulation of FA synthesis. Thus, our data demonstrate that PTCH also controls intracellular cholesterol levels in $C$. elegans. Moreover, we show that PTCH thereby impinges on FA metabolism, organellar structure and fat storage capacity. 


\section{Results}

\section{PTC-3 has cell autonomous and non-autonomous functions and is required for lipid storage in the intestine.}

In order to understand the function of PTCH proteins in C. elegans, we decided first to revisit the phenotypes caused by the depletion of the somatically expressed PTC-3. Like its mammalian homolog, ptc-3 is essential for development. Consistently, it has been reported that ptc-3(RNAi) results in growth, molting and vulva morphogenesis defects ${ }^{17,18}$. Given the essential role of PTCH in development, we started the knockdown by RNAi only at the L2 stage of development, allowing the worms to progress further in development and even some to reach adulthood. In addition to the previously reported phenotypes, we noticed that the ptc-3(RNAi) animals were much paler than their mock-treated counterparts (Fig. 1A). Pale worms are an indication for defects in fat storage. C. elegans has a much simpler body plan than humans and hence some $C$. elegans organs take over more functions. For example, the worm intestine has paracrine functions, and also serves also as the fat storage organ ${ }^{25}$. Thus, in a simplified view, the $C$. elegans intestine represents the functional equivalent of the human intestine, adipose tissue and liver. To test whether PTC-3 was expressed in the gut as indicated by genome-wide expression analyses ${ }^{20}$, we raised antibodies against PTC-3 (Fig. S1A). Those antibodies decorated the apical membrane of gut epithelial cells, while no plasma membrane signal was detected in oocytes, consistent with the notion that PTC-3 is present only in somatic tissues (Fig. 1B). This localization was confirmed with a GFP-tagged PTC-3 (Fig. S1B).

To determine which phenotype is dependent on intestinal PTC-3, we performed a gut-specific knockdown of PTC-3 ${ }^{26}$. ptc-3(RNAjut) animals were still paler and thinner than mock-treated animals (Fig. 1C). Moreover, vulva morphogenesis defects were also observed upon the ptc-3(RNAj $\left.{ }^{\text {gut }}\right)$ regime, indicating that PTC-3 has cell autonomous and non-autonomous functions.

As outlined above, pale phenotypes are often associated with lipid storage defects in worms ${ }^{25,27}$. Nile Red staining indeed showed a reduction in lipid content in ptc-3(RNAi) animals (Fig. 1D and E). Of note, this reduction was observed in the intestine, but not in the germline, in accordance with the absence of PTC-3 expression in oocytes. A drawback of Nile Red staining is that autofluorescence in the intestine 
bioRxiv preprint doi: https://doi.org/10.1101/816256; this version posted October 23,2019 . The copyright holder for this preprint (which was not certified by peer review) is the author/funder, who has granted bioRxiv a license to display the preprint in perpetuity. It is made available under aCC-BY-ND 4.0 International license.

Figure 1

A

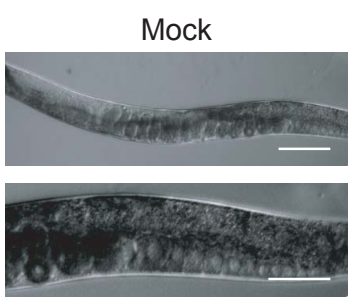

C

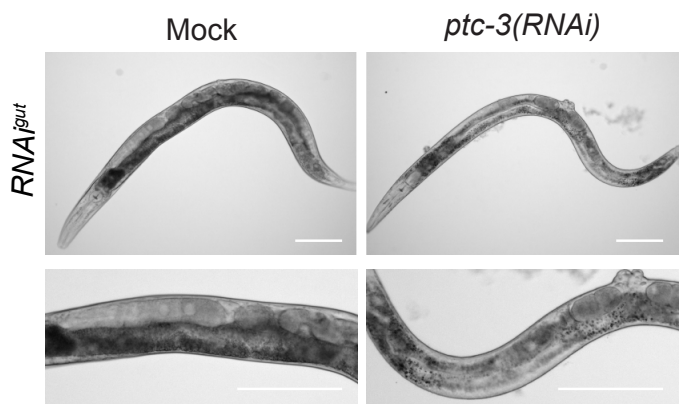

$\mathrm{E}$

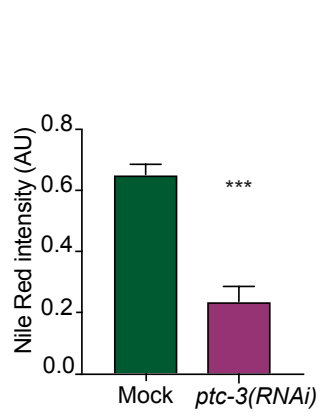

$\mathrm{F}$

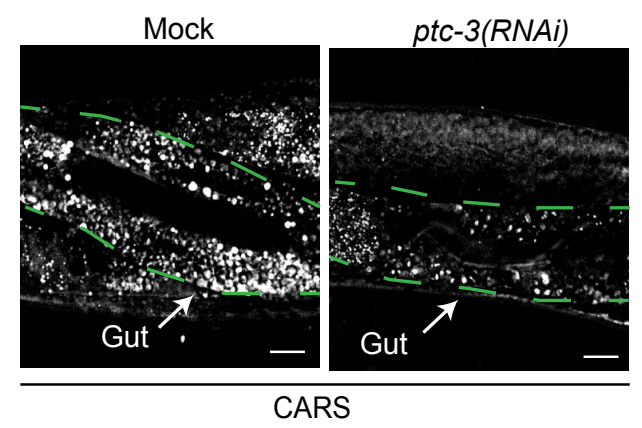

PTC-3

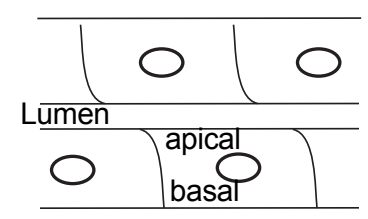

Gut

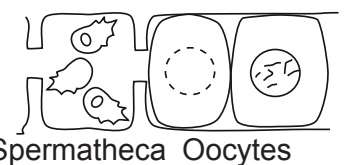

D

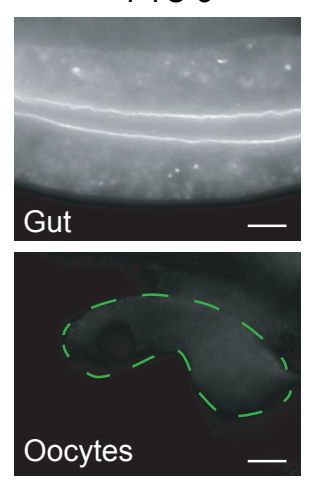

ptc-3(RNAi)

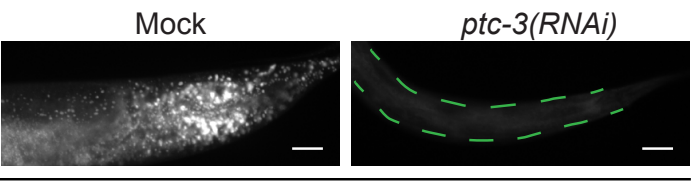

Nile Red

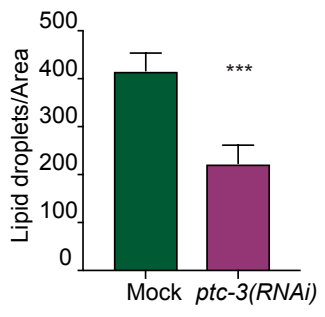


bioRxiv preprint doi: https://doi.org/10.1101/816256; this version posted October 23, 2019. The copyright holder for this preprint (which was not certified by peer review) is the author/funder, who has granted bioRxiv a license to display the preprint in perpetuity. It is made available under aCC-BY-ND 4.0 International license.

\section{Figure S1}

A

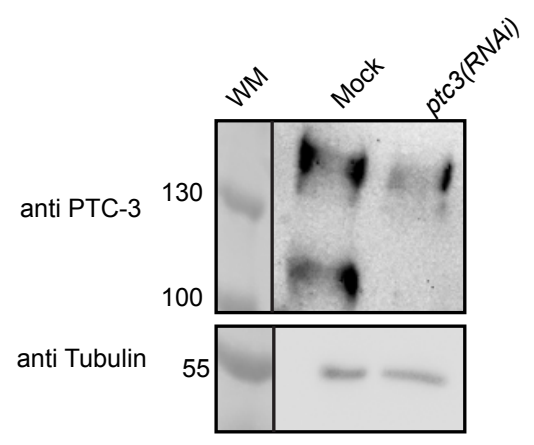

B

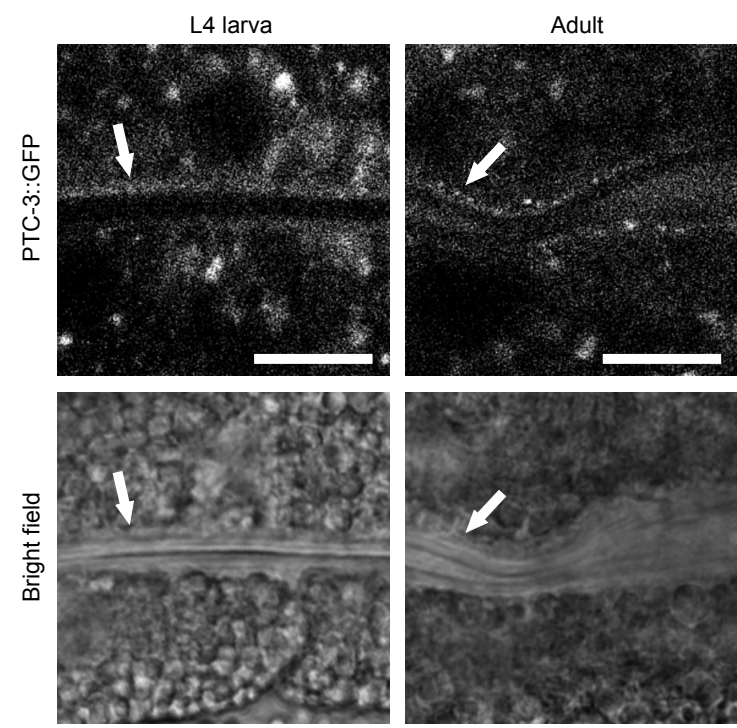

C
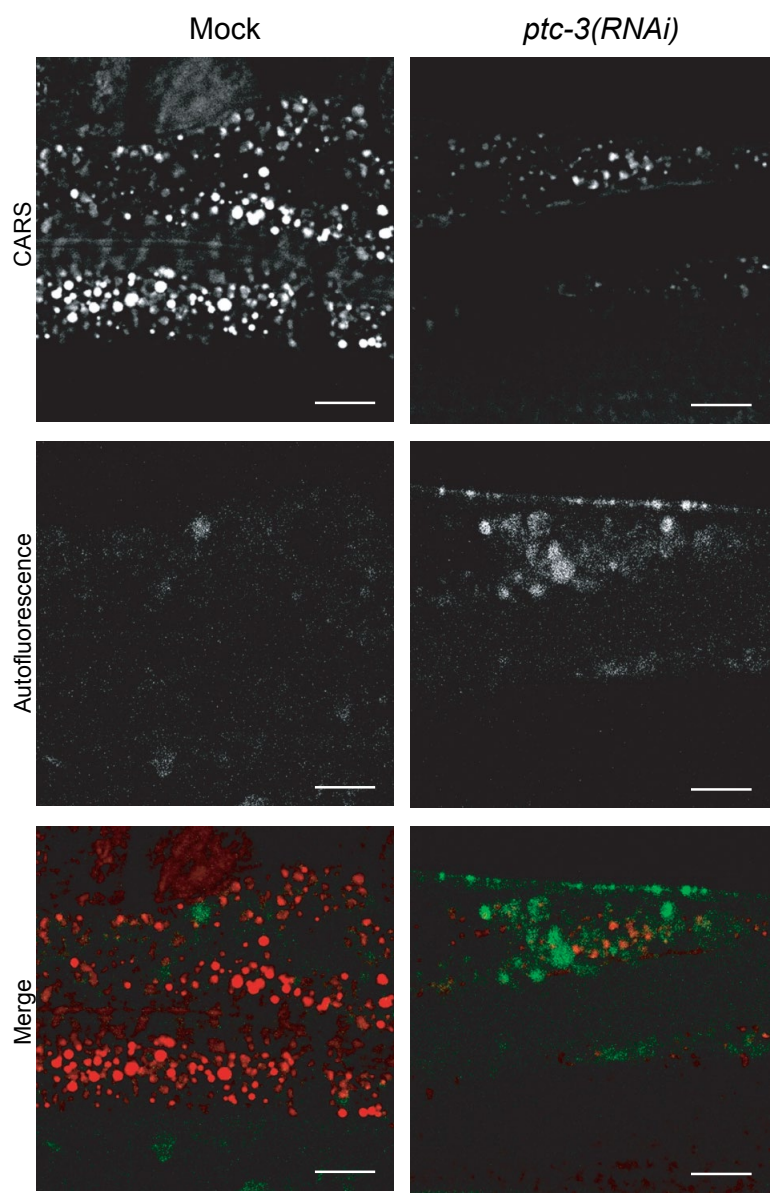
caused by lysosome-related organelles (LROs) is also potentially measured at the same time, which may confound the results. Therefore, we turned to Coherent antiStokes Raman Scattering Microscopy (CARS), a dye-free method recognized for accurate in vivo lipid detection in worms ${ }^{28}$. This analysis confirmed the Nile Red staining, and we observed an about 50\% reduction in lipid content (Fig. 1F and G), indicating that we can use Nile Red for further analysis. The CARS signal did not overlap with the autofluorescence of LROs (Fig. S1C). We conclude that loss of PTC3 causes a reduction in fat storage in the intestine.

\section{PTC-3 is a cholesterol transporter}

Recent data suggested that mammalian PTCH1 acts as a cholesterol transporter 1,5,6,8-10. To investigate, whether PTC-3 shares the function of PTCH1 as cholesterol transporter, we first expressed PTC-3 in Saccharomyces cerevisiae, which does not contain any cholesterol ${ }^{29}$ and measured cholesterol efflux from cells using TopFluor cholesterol in pulse-chase experiment (Fig. 2A). PTC-3 expressing yeast cells exported cholesterol significantly faster out of the cell than control cells, similar to what has been observed for mammalian $\mathrm{PTCH} 1^{5}$. This efflux capacity was dependent on an active permease domain, since a mutation in the permease domain ${ }^{18}, p t c-3^{D 697 A}$, strongly reduced the cholesterol efflux. The ptc-3 $3^{D 697 A}$ mutation has been reported to cause larval lethality in worms ${ }^{18}$, establishing that cholesterol efflux is the essential function of PTCH. Next, we repeated the pulse-chase experiment in worms. While in mock treated worms, TopFluor cholesterol was present mostly in the gut lumen, it was still strongly accumulated in the intestine in ptc-3(RNAi) worms after the washout, further demonstrating the role as cholesterol transporter (Fig. 2B). Finally, we measured sterol levels by mass spectrometry. Cholesterol levels were increased in ptc-3(RNAi) worms, while 7-dihydrocholesterol (7-DHC) and lophenol levels were decreased (Fig. 2C). 7-DHC and lophenol are downstream products of cholesterol in worms, indicating that cholesterol metabolism might also be affected by ptc-3(RNAi). Taken together our data strongly suggest that PTC-3, like PTCH1, is a cholesterol transporter at the plasma membrane. 
bioRxiv preprint doi: https://doi.org/10.1101/816256; this version posted October 23, 2019. The copyright holder for this preprint (which was not certified by peer review) is the author/funder, who has granted bioRxiv a license to display the preprint in perpetuity. It is made available under aCC-BY-ND 4.0 International license.

Figure 2

A

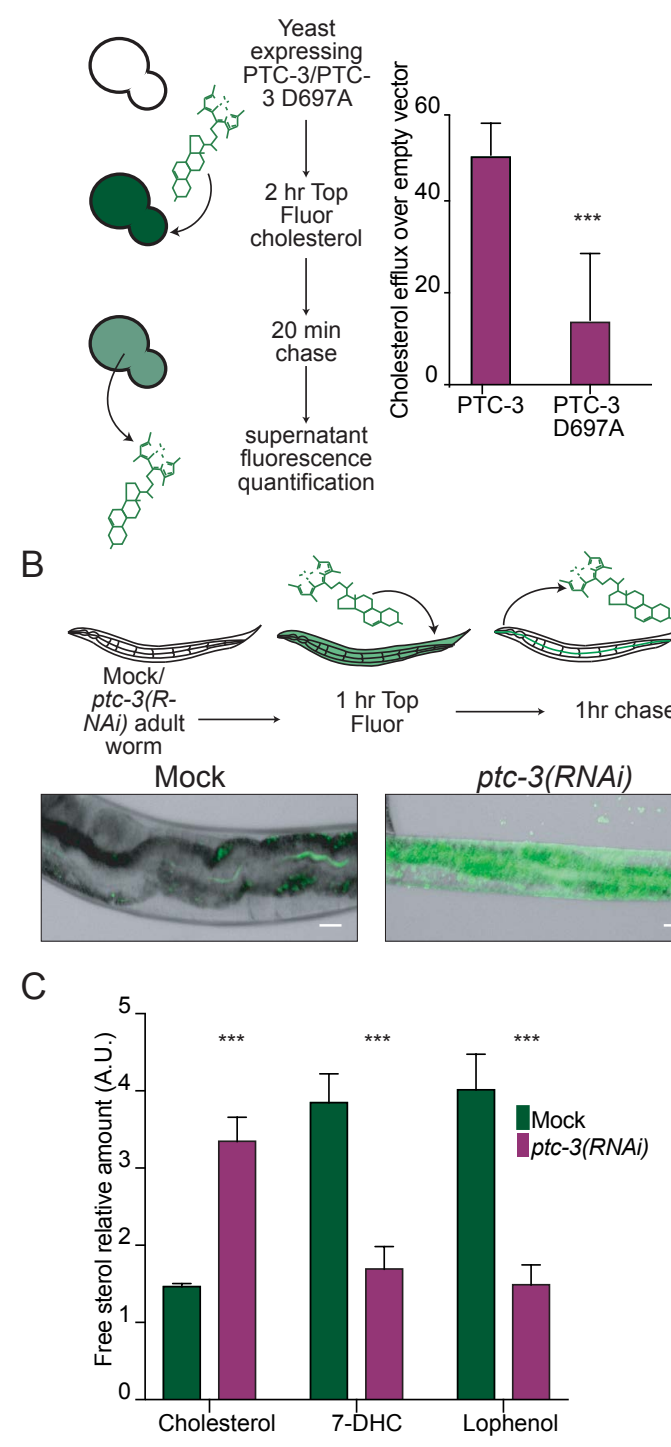

$\mathrm{D}$
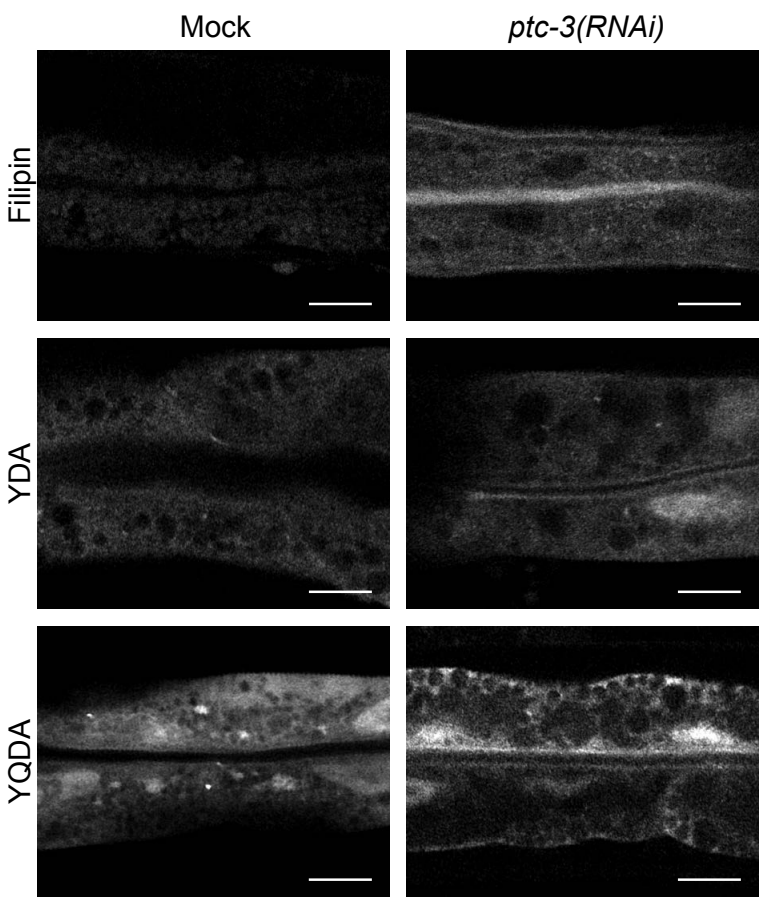

$\mathrm{E}$
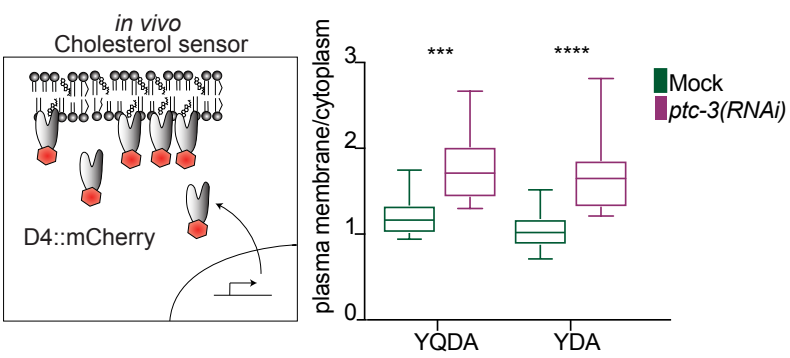


\section{Cholesterol accumulates predominantly in the apical membrane in the intestine of ptc-3(RNAi) animals}

Cholesterol accumulates in ptc-3(RNAi) worms because it cannot be pumped out of the cells. In addition, cholesterol is not efficiently metabolized into 7-DHC and lophenol under those conditions. We speculated where the excess of cholesterol would reside in the cell. First, we used filipin, which binds specifically to cholesterol. While we could barely detect any filipin staining in mock-treated animals, ptc-3(RNAi) worms showed a strong fluorescent signal in the apical membrane in the intestine and also some appreciable increase in intracellular fluorescence (Fig. 2D). To corroborate this finding, we next employed two versions of the domain 4 of perfringolysin fused to mCherry probe (D4-mCherry), YDA and YQDA, which have different sensitivities in the detection of cholesterol ${ }^{30-32}$. We expressed the probes constitutively in the $C$. elegans intestine and analyzed their cellular distribution. Similar to the filipin staining, the $\mathrm{mCherry} \mathrm{signal} \mathrm{increased} \mathrm{in} \mathrm{the} \mathrm{plasma} \mathrm{membrane} \mathrm{for} \mathrm{both} \mathrm{probes} \mathrm{in} \mathrm{ptc-3(RNAi)}$ animals compared to mock treated controls. (Fig. 2D and E). Thus, the strongest cholesterol accumulation is observed in the apical membrane in the intestine of ptc3(RNAi) animals. We envisage that cholesterol levels are also increased, albeit to a lesser extent, in intracellular membranes.

\section{Low dietary cholesterol rescues ptc-3(RNAi) phenotypes}

It is plausible that the cholesterol accumulation is the cause for the observed phenotypes in ptc-3(RNAi) animals. C. elegans is unable to synthesize cholesterol and must ingest it through the diet ${ }^{33}$. In the lab, cholesterol is provided in the growth medium. Strikingly, when we omitted cholesterol from the growth medium, ptc-3(RNAi) worms developed much better, with 89\% reaching adulthood (Fig. $3 A$ and B). Moreover, the pale phenotype was strongly reduced, and lipid storage was improved (Fig. 3C and D). Thus, reducing cholesterol accumulation rescued developmental as well as fat storage defects in ptc-3(RNAi) worms. Since cholesterol conversion into 7DHC was also impaired, we asked whether increasing the levels of 7-DHC would likewise rescue the ptc-3(RNAi) phenotypes. However, addition of 7-DHC did not alleviate the ptc-3(RNAi) phenotype (Fig. S2). We conclude that most of the ptc3(RNAi) phenotypes are linked to the regulation of intracellular cholesterol levels. Moreover, the accumulation of cholesterol, and not the inability of the ptc-3(RNAi) animals to process cholesterol efficiently, appears to be detrimental for the organism. 
bioRxiv preprint doi: https://doi.org/10.1101/816256; this version posted October 23, 2019. The copyright holder for this preprint (which was not certified by peer review) is the author/funder, who has granted bioRxiv a license to display the preprint in perpetuity. It is made available under aCC-BY-ND 4.0 International license.

\section{Figure 3}

A
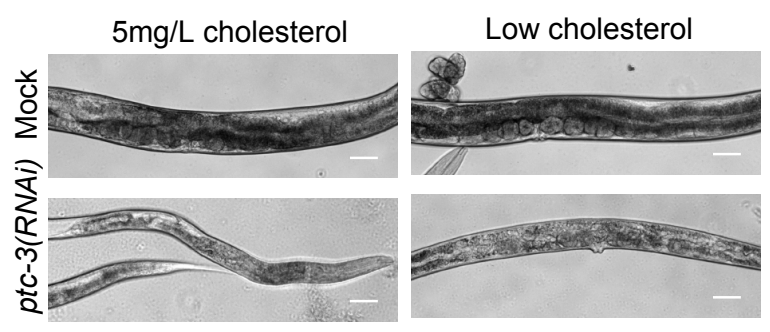

\section{C}
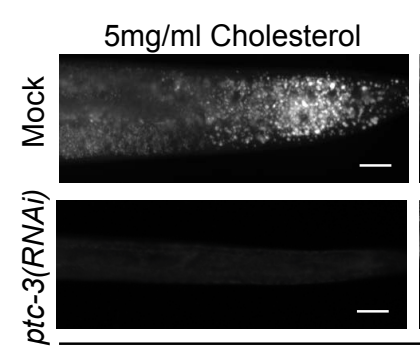

Nile Red
B

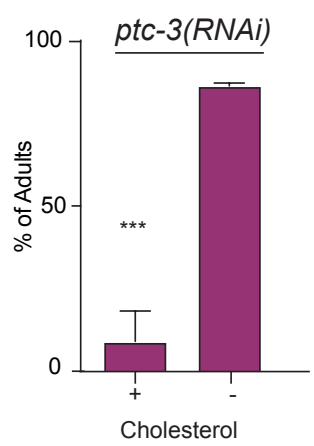

D

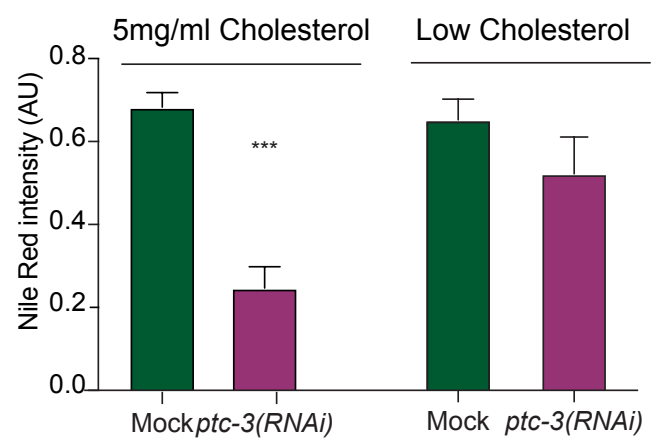


bioRxiv preprint doi: https://doi.org/10.1101/816256; this version posted October 23, 2019. The copyright holder for this preprint (which was not certified by peer review) is the author/funder, who has granted bioRxiv a license to display the preprint in perpetuity. It is made available under aCC-BY-ND 4.0 International license.

\section{Figure S2}

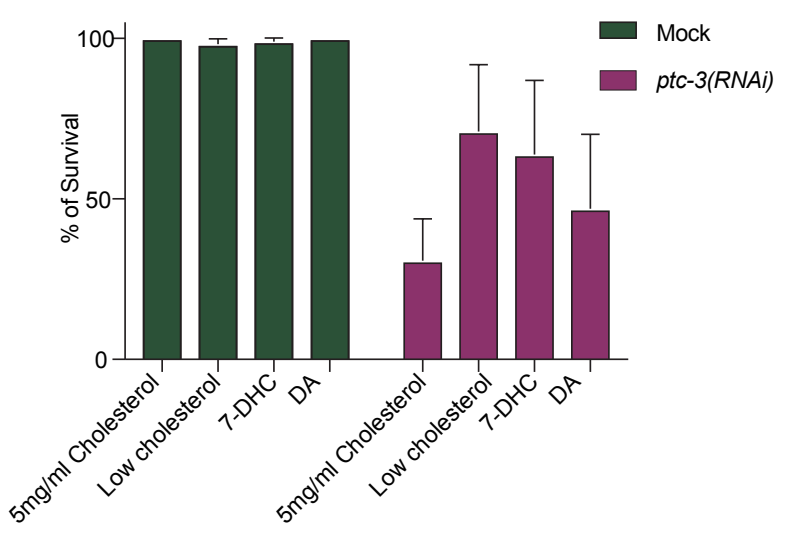




\section{Lipid droplet biogenesis and ER morphology are impaired upon ptc-3(RNAi)}

Cellular fat is mostly stored in lipid droplets, which originate from the endoplasmic reticulum (ER). In ptc-3(RNAi) animals, we observed defects in fat storage dependent on the intracellular cholesterol levels. Thus, we investigated whether the ER was affected by loss of PTC-3 function using intestinally expressed TRAM-GFP. We observed morphological alterations in the ER in ptc-3(RNAi) animals, which were, however, hard to interpret (Fig. S3). To gain a better understanding of the phenotype, we performed electron microscopy. Not unexpectedly, given the fat storage defect, lipid droplets were essentially absent in ptc-3(RNAi) intestines (Fig 4A). Even more strikingly, the ER had lost most of its reticulate structures and formed long lines. Such long lines in 2D are indicative of ER sheets in $3 D^{34}$. We used focused ion beam scanning electron microscopy (FIB-SEM) and machine learning algorithms to obtain information on the ER structure in $3 \mathrm{D}$. Indeed, the reticulate, tubular structure of the ER was dramatically reduced in ptc-3(RNAi) when compared to mock; instead enormous ER-sheets and clusters were formed (Fig. 4B, Fig. S4, movies S1 and S2). Taken together, our data so far suggest that the cholesterol accumulation, due to the absence of PTC-3, impairs ER structure and thereby lipid droplet formation. If the cellular cholesterol levels were indeed the critical factor, then reducing dietary cholesterol in ptc-3(RNAi) animals should alleviate the ER phenotype. Indeed, ptc3(RNAi) animals raised on low cholesterol diet displayed reticulated ER and lipid droplets (Fig. 4A). Thus, cellular cholesterol levels strongly influence ER morphology and function. At this point we were unable to determine whether this effect is direct or indirect. Even though, most of the cholesterol accumulated in the apical plasma membrane in ptc-3(RNAi) animals, we cannot exclude, that there is also an accumulation of cholesterol in the ER. Unfortunately, filipin bleaches very fast, and the D4-mCherry sensors are present throughout the cell, so that we are only be able to detect very strong local accumulations. Still, the inability of the ER membrane to form lipid droplets and the sheet structure might be linked to the increased membrane bending rigidity.

\section{Fatty acid acyl chain length and desaturation is reduced in ptc-3(RNAi) animals}

To test this hypothesis, we first performed a simple experiment, in which we modulated the growth temperature. Membrane fluidity increases as a function of temperature, while membrane bending rigidity decreases. Consistent with our hypothesis, the 
bioRxiv preprint doi: https://doi.org/10.1101/816256; this version posted October 23, 2019. The copyright holder for this preprint (which was not certified by peer review) is the author/funder, who has granted bioRxiv a license to display the preprint in perpetuity. It is made available under aCC-BY-ND 4.0 International license.

\section{Figure S3}
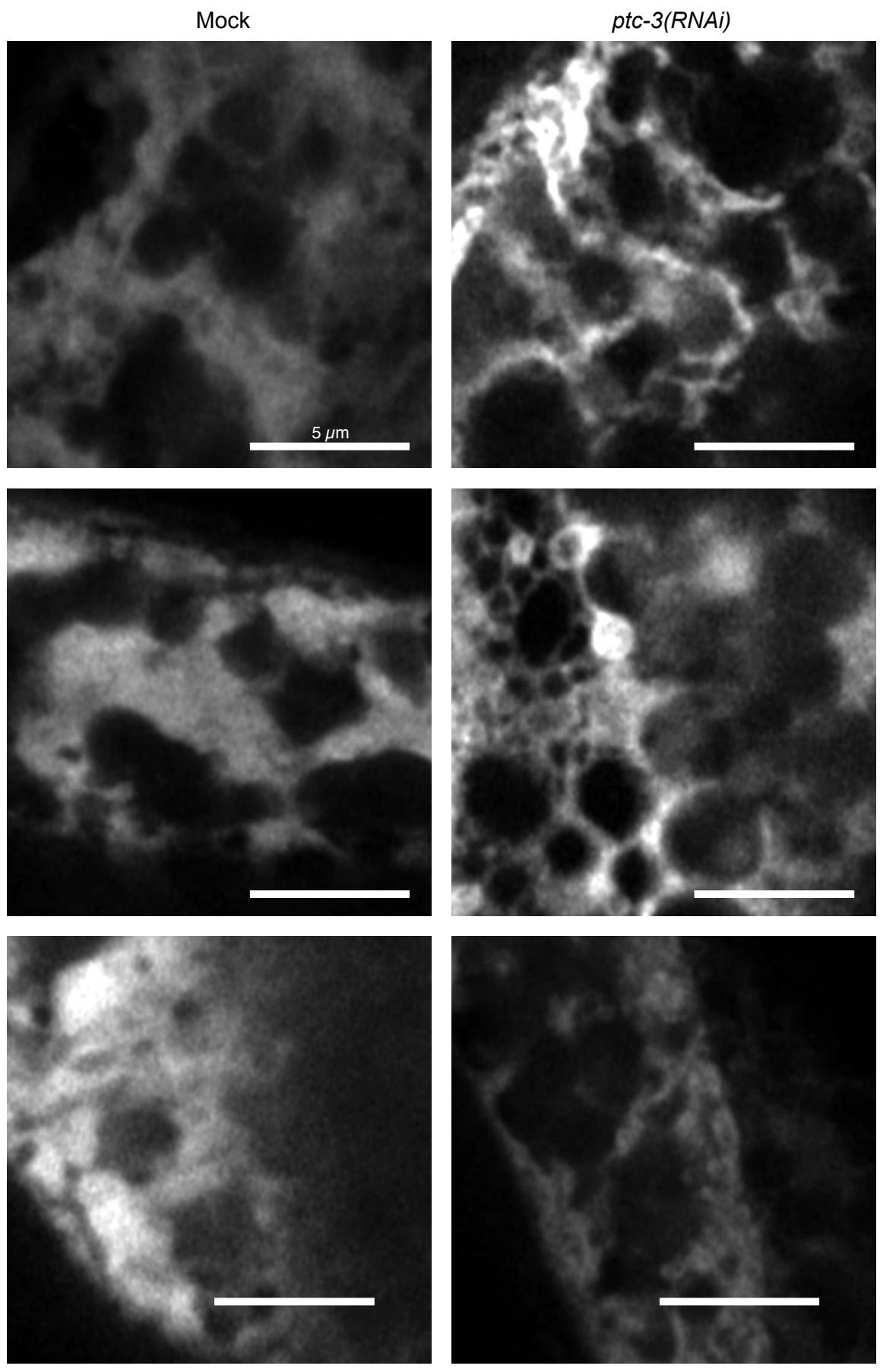
bioRxiv preprint doi: https://doi.org/10.1101/816256; this version posted October 23, 2019. The copyright holder for this preprint (which was not certified by peer review) is the author/funder, who has granted bioRxiv a license to display the preprint in perpetuity. It is made available under aCC-BY-ND 4.0 International license.

Figure 4

A
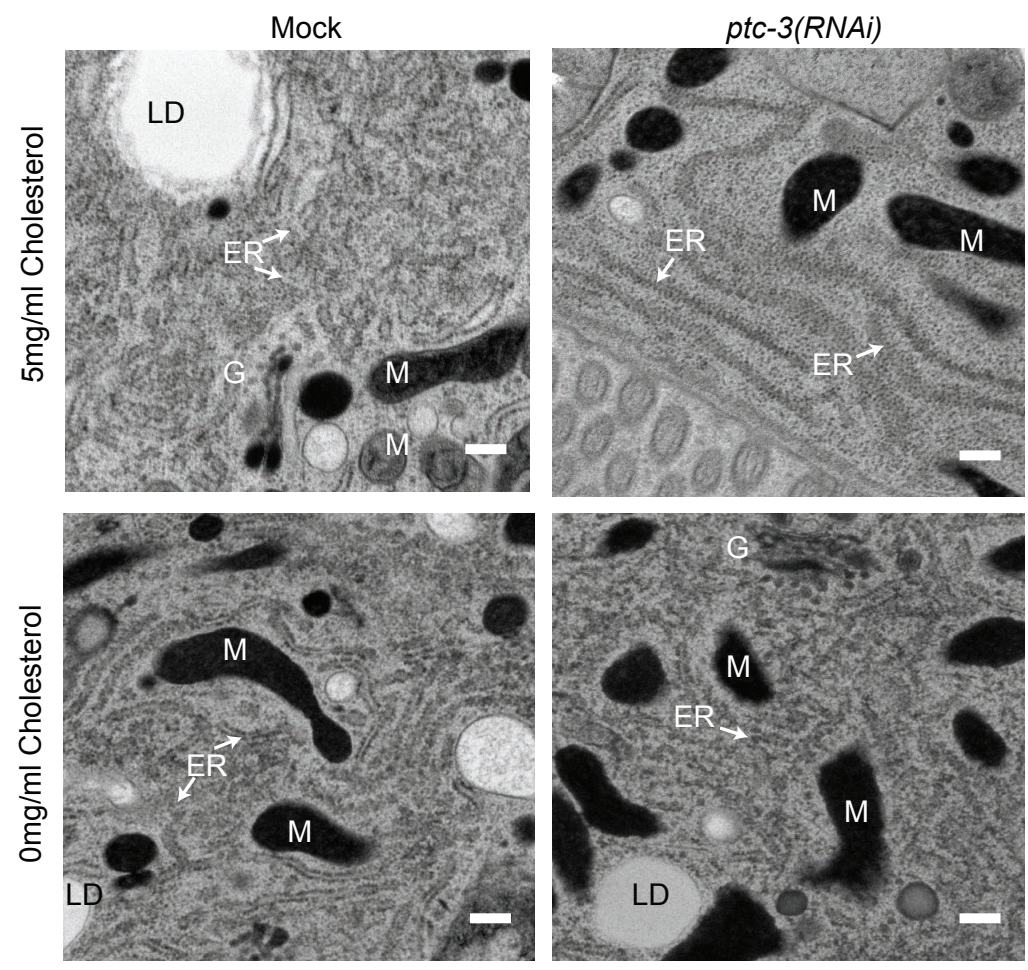

B
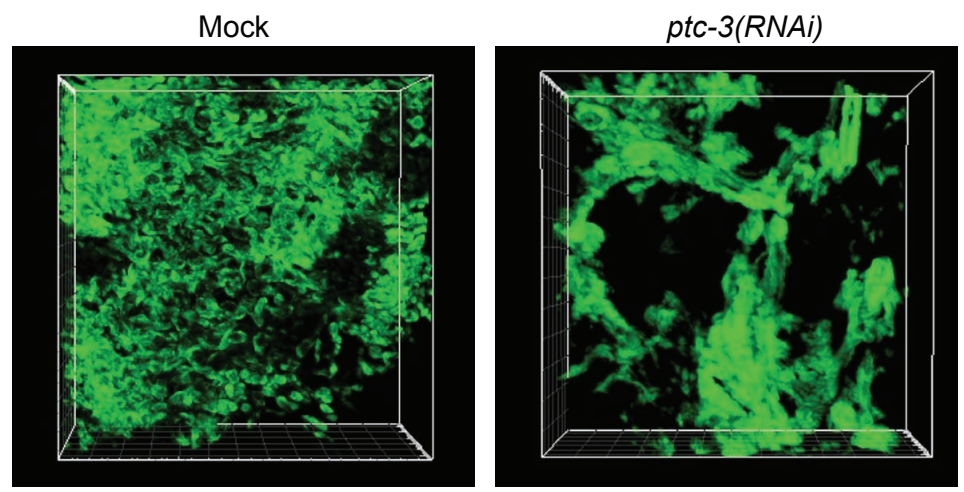
bioRxiv preprint doi: https://doi.org/10.1101/816256; this version posted October 23, 2019. The copyright holder for this preprint (which was not certified by peer review) is the author/funder, who has granted bioRxiv a license to display the preprint in perpetuity. It is made available under aCC-BY-ND 4.0 International license.

\section{Figure S4}
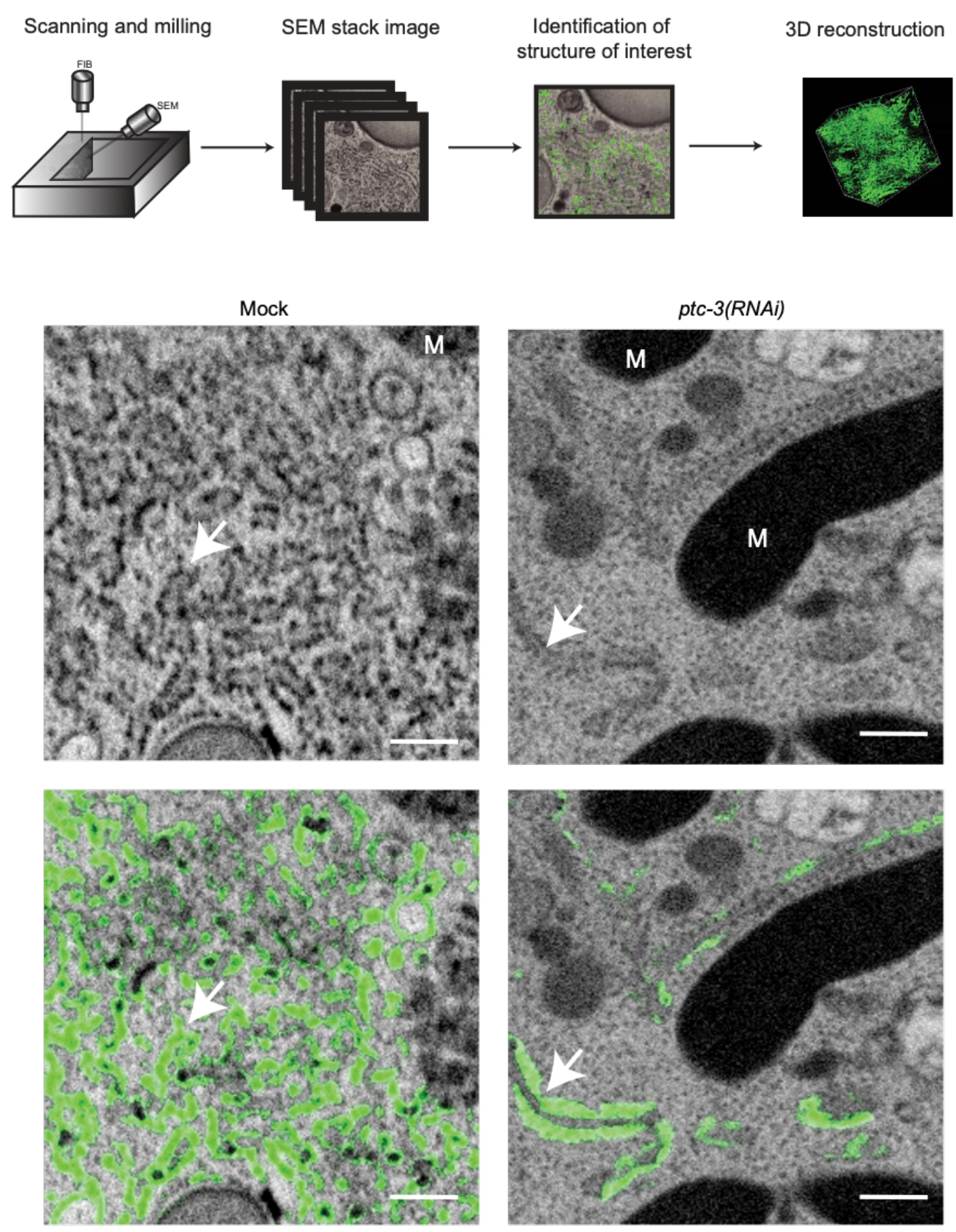
development and viability of ptc-3(RNAi) animals were improved at an elevated temperature (Fig. 5A and B).

Another factor, which determines the stiffness or fluidity of membranes is the saturation of the acyl chains of lipids. Saturated acyl chains are considered to be relatively straight, allowing a high packing rate of lipids accompanied with the generation of an ordered phase and a reduction in fluidity. In contrast, desaturated fatty acids correlate with less dense packing, higher membrane fluidity and lower bending rigidity. Therefore, we performed lipidomics and determined the level of phospholipid acyl chain saturation upon ptc-3(RNAi). We did not observe any major difference in the headgroup composition of the most important lipid species (Fig. 5C). In contrast, we detected a reduction in polyunsaturated fatty acids (PUFAs) in ptc$3(R N A i)$ worms as there was a marked decrease in acyl chain length and desaturation (Fig. 5D). This reduction in PUFAs is not due to a general reduction in lipids upon ptc3(RNAi) compared to mock treatment, but rather reflects a shift from PUFAs to more saturated, shorter FAs. This shift towards more saturated FAs supports our hypothesis that the cholesterol accumulation contributes, directly or indirectly, to the morphological changes of the ER membrane.

\section{NHR-49 and FAT-7 overexpression rescue ptc-3(RNAi) animals}

The reduction in PUFAs could potentially be due to inhibition or lower expression of fatty acid desaturases and elongases. A potential candidate to check this hypothesis is the desaturase FAT-7, which appeared to be down-regulated during heat adaptation to counteract the increase in membrane fluidity at high temperature ${ }^{35}$. Overexpression of FAT-7 in the intestine resulted in better survival of ptc-3(RNAi) animals (Fig. 6A and $B$ ). The rescued animals were darker than their counterparts (Fig. 6A), suggesting that they were able to store fat. FAT-7 expression is regulated by the PPAR $\alpha$ homolog NHR-49 ${ }^{36,37}$. Similar to what we had observed for FAT-7 overexpression, increasing intestinal NHR-49 levels improved survival of ptc-3(RNAi) animals (Fig. 6A and B). Rescue of survival due to NHR-49 overexpression was accompanied by restoration of fat storage (Fig. 6A-D), suggesting that NHR-49 is a major downstream effector of PTC-3. NHR-49 partners with NHR-80, a homolog of mammalian HNF4, to regulate fatty acid desaturation ${ }^{36}$. However, overexpression of NHR-80 did not rescue the ptc- 
bioRxiv preprint doi: https://doi.org/10.1101/816256; this version posted October 23, 2019. The copyright holder for this preprint (which was not certified by peer review) is the author/funder, who has granted bioRxiv a license to display the preprint in perpetuity. It is made available under aCC-BY-ND 4.0 International license.

Figure 5

A

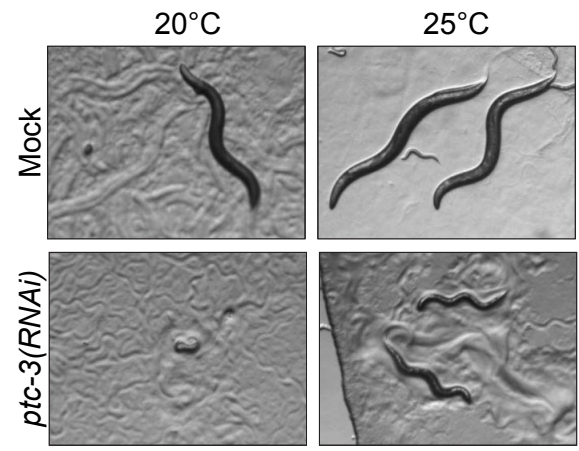

B

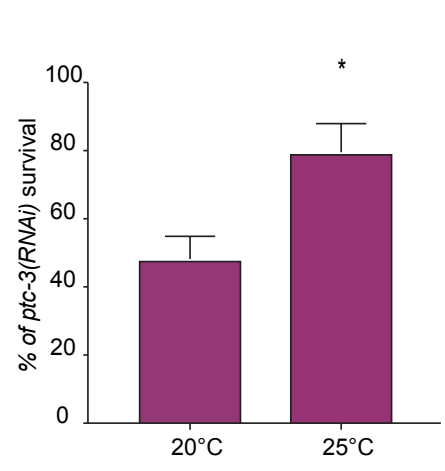

C

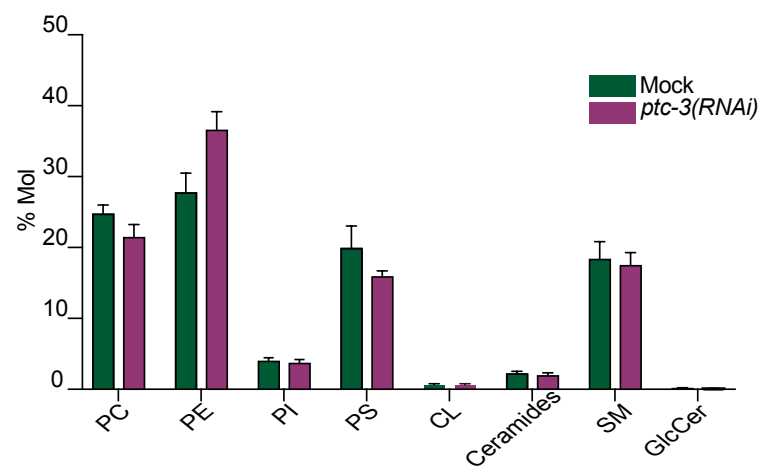

D

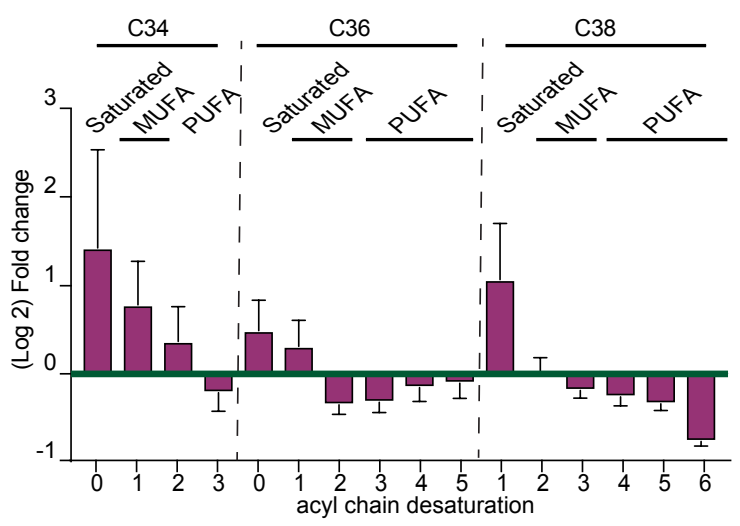


bioRxiv preprint doi: https://doi.org/10.1101/816256; this version posted October 23, 2019. The copyright holder for this preprint (which was not certified by peer review) is the author/funder, who has granted bioRxiv a license to display the preprint in perpetuity. It is made available under aCC-BY-ND 4.0 International license.

Figure 6

A

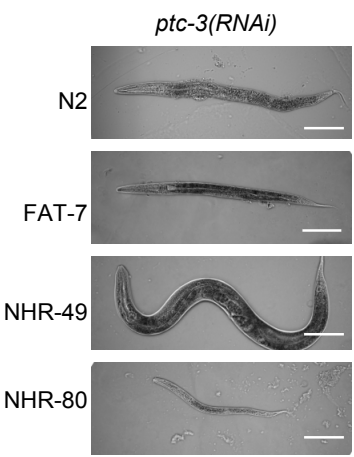

B

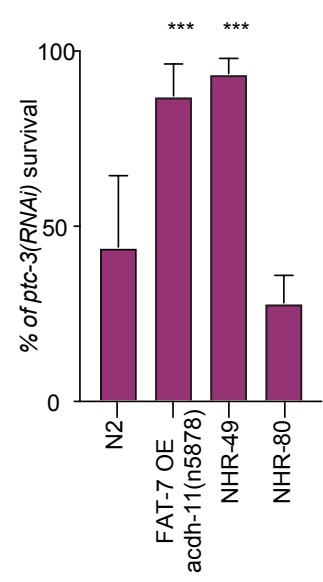

C

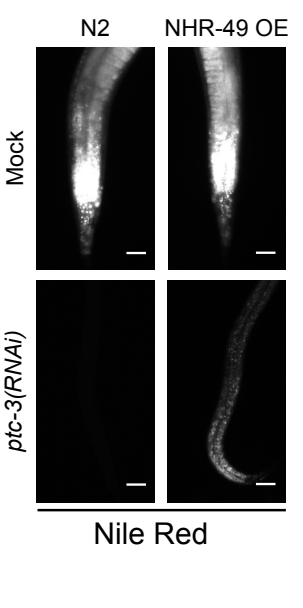

D

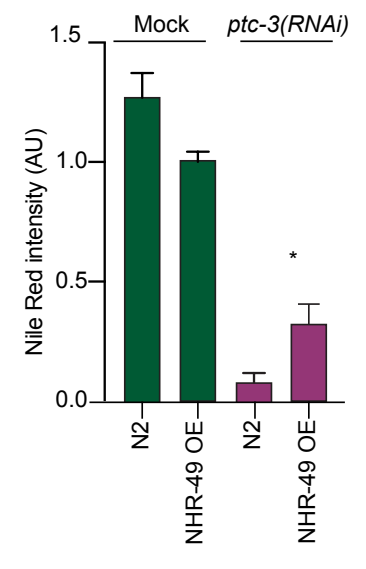

E

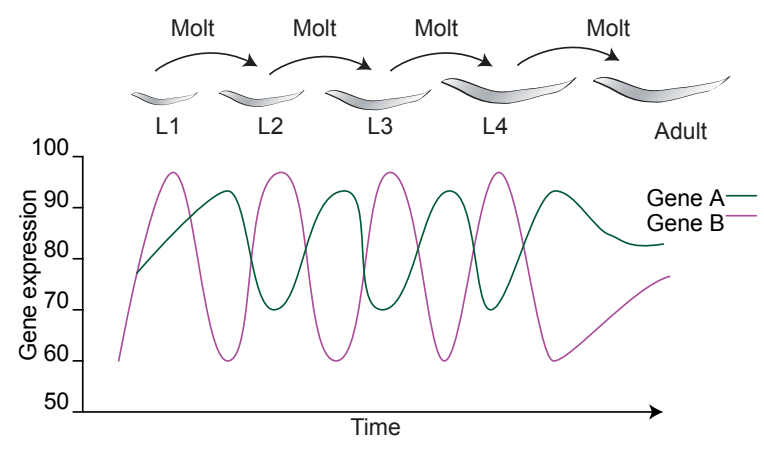

G
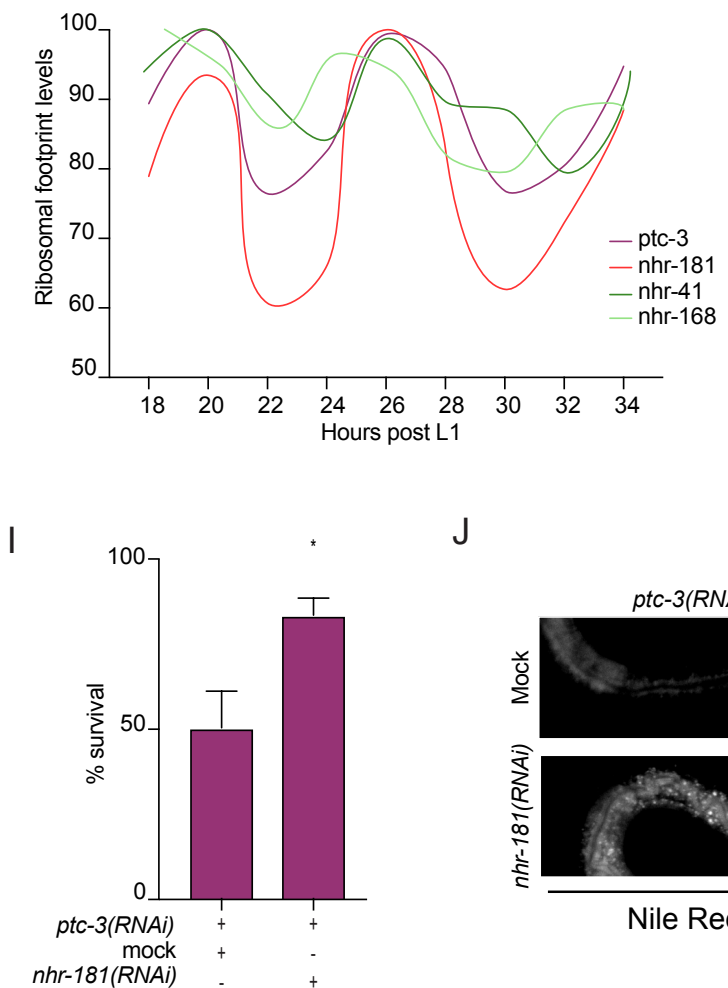

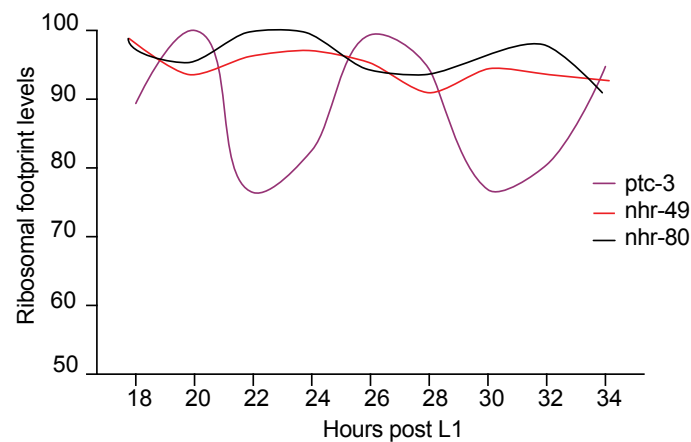

H

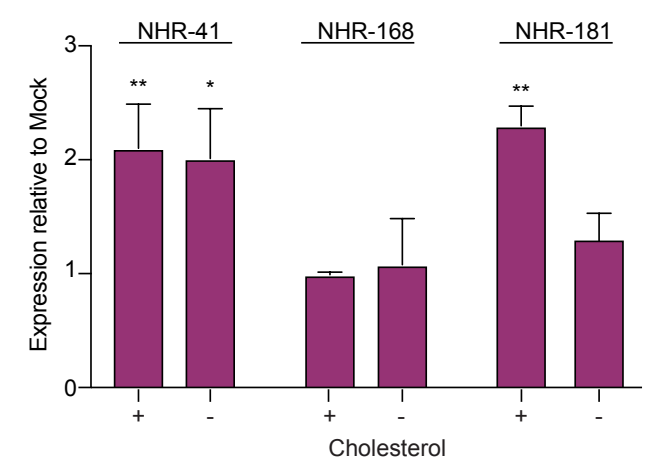

K

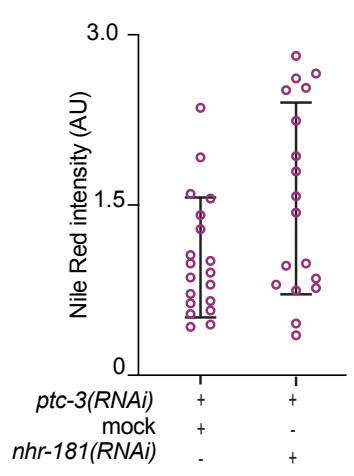


3(RNAi) phenotype (Fig. 6A and B). Our data are consistent with the notion that NHR49 and FAT-7 are modulators of membrane bending rigidity.

\section{Loss of NHR-181 rescues the high cholesterol induced phenotypes in ptc-} 3(RNAi) animals

Nuclear hormone receptors often act context-dependent. Therefore, we wondered, whether other NHRs or the loss thereof may contribute to the ptc-3(RNAi) phenotype. NHR-8, the C. elegans ortholog of vertebrate liver $X$ and vitamin D receptors, was also shown to influence cholesterol levels and fat content ${ }^{38,39}$. Since $n h r-8(R N A i)$ animals contained more fat ${ }^{39}$, we speculated whether loss of NHR-8 could rescue the ptc3(RNAi) phenotype. However, we could not detect any rescue (Fig. S5A). This result may not have been so unexpected since $n h r-8$ mutants contain less unsaturated fatty acids ${ }^{39}$. Overexpression of NHR-8 still did not alleviated ptc-3(RNAi) defects (Fig. S5B), indicating that NHR-8 and PTC-3 act independently.

C. elegans expresses 278 nuclear hormone receptors. To identify possible NHRs important in a PTC-3-dependent pathway, we turned to genome-wide expression data during development. $C$. elegans goes through 4 larval stages before reaching adulthood (Fig. 6E). Each transition from one larval stage to the next is accompanied by the synthesis of a new, larger cuticula, in a process referred to as molting. Genome-wide RNAseq and Riboseq throughout C. elegans development revealed an oscillatory behavior of gene expression for many genes ${ }^{40}$ (Fig. 6E). Given the general role of PTC-3 in development and the observed cuticle defects upon ptc3(RNAi), it was not surprising to find that PTC-3 expression also oscillated (Fig. 6F). However, NHR-49 expression remained constant during development (Fig. 6F). We then asked, which other NHRs would oscillate in a similar manner as PTC-3. Three NHRs emerged as possible candidates: NHR-41, NHR-168 and NHR-181 (Fig. 6G). We hypothesized that the expression levels of the NHRs should be responsive to cholesterol levels. Of the three, only expression levels of the HNF4 homolog NHR-181 were upregulated in high cholesterol i.e. ptc-3(RNAi), and reduced under low cholesterol conditions (Fig. 6H). More importantly, knockdown of NHR-181 rescued the ptc-3(RNAi) induced lethality to a similar extent than overexpression of NHR-49, irrespective of the cholesterol present in the medium (Fig. 6B and I). Moreover, fat content was restored to a similar extent (Fig. $6 \mathrm{~J}$ and $\mathrm{K}$, compare $\mathrm{J}$ and $\mathrm{C}$ ). Taken 
bioRxiv preprint doi: https://doi.org/10.1101/816256; this version posted October 23, 2019. The copyright holder for this preprint (which was not certified by peer review) is the author/funder, who has granted bioRxiv a license to display the preprint in perpetuity. It is made available under aCC-BY-ND 4.0 International license.

Figure S5

A

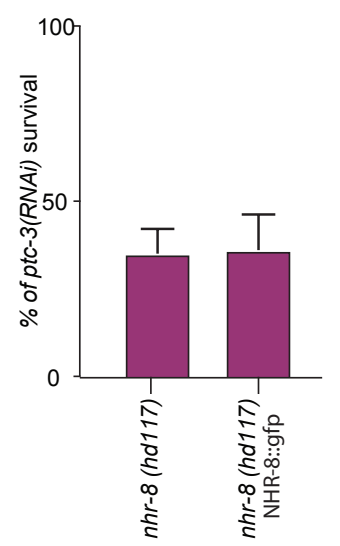

B

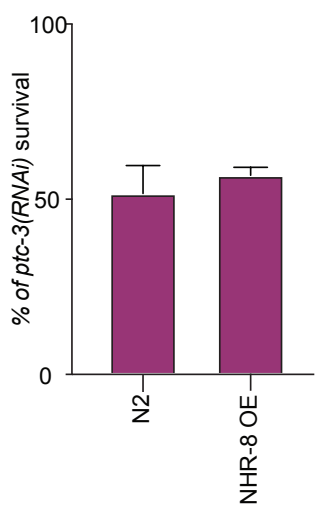

C

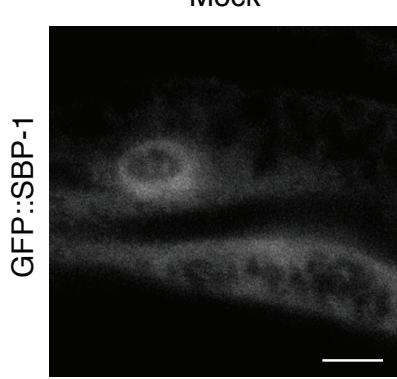

ptc-3(RNAi)

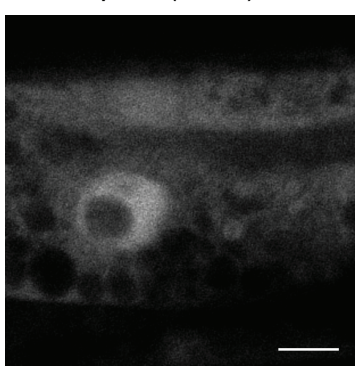

Mock

Low cholestrol

ptc-3(RNAi)

Low cholestrol

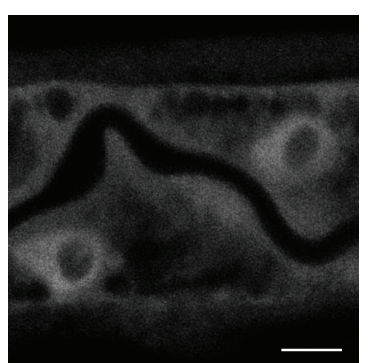

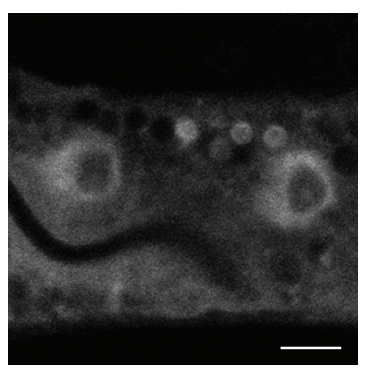


together, our data imply that NHR-49 positively and NHR-181 negatively regulate membrane bending properties and fat storage in response to high cholesterol levels.

\section{Discussion}

We explored the role of the $C$. elegans PTCH homolog, PTC-3, in the absence of the classical functional hedgehog signaling pathway. The function of PTCH proteins is conserved from $C$. elegans to man because similar to what has been proposed for mammalian PTCH ${ }^{1,6,41}$, PTC-3 is a cholesterol transporter, which exports cholesterol out of the cell. PTC-3 appears to be the major cholesterol transporter in the apical plasma membrane in the $C$. elegans intestine, since knock-down of PTC-3 resulted in strong intracellular cholesterol accumulation, most notably in the apical plasma membrane.

As a consequence of the cholesterol accumulation, the balance of tubular and sheet-like ER was strongly skewed towards sheet structures (Fig. 7). Moreover, lipid droplet synthesis, which originates at the ER was greatly reduced. We propose that the ER membranes in ptc-3(RNAi) animals have an increased bending rigidity, which does not allow bulging out of lipid droplets. Whether triglycerides still accumulate within the lipid bilayer remains unclear because their detection in the ER membrane was not possible. However, we observed an increase in saturated and monounsaturated fatty acid acyl chains (MUFAs) at the expense of polyunsaturated fatty acids (PUFAs) upon knockdown of PTC-3. This imbalance towards shorter and saturated acyl chains should also increase lipid packing and increase membrane bending rigidity. This effect would then be intensified by the accumulation of cholesterol in intracellular membranes, which would furthermore promote membrane stiffness.

While the increase of membrane bending rigidity is probably sufficient to inhibit lipid droplet formation, vesicle formation at the ER and the Golgi apparatus may not be as strongly affected by cholesterol accumulation and the increase in MUFAs because we still observed stacked Golgi apparatus by electron microscopy (Fig S6). The difference between lipid droplet formation and vesicle budding is that the COPII coat can bend the entire lipid bilayer ${ }^{42}$, while the triglycerides must deform the membrane from within and push the lipid bilayer apart, a process, which might be less 
bioRxiv preprint doi: https://doi.org/10.1101/816256; this version posted October 23, 2019. The copyright holder for this preprint (which was not certified by peer review) is the author/funder, who has granted bioRxiv a license to display the preprint in perpetuity. It is made available under aCC-BY-ND 4.0 International license.

Figure 7

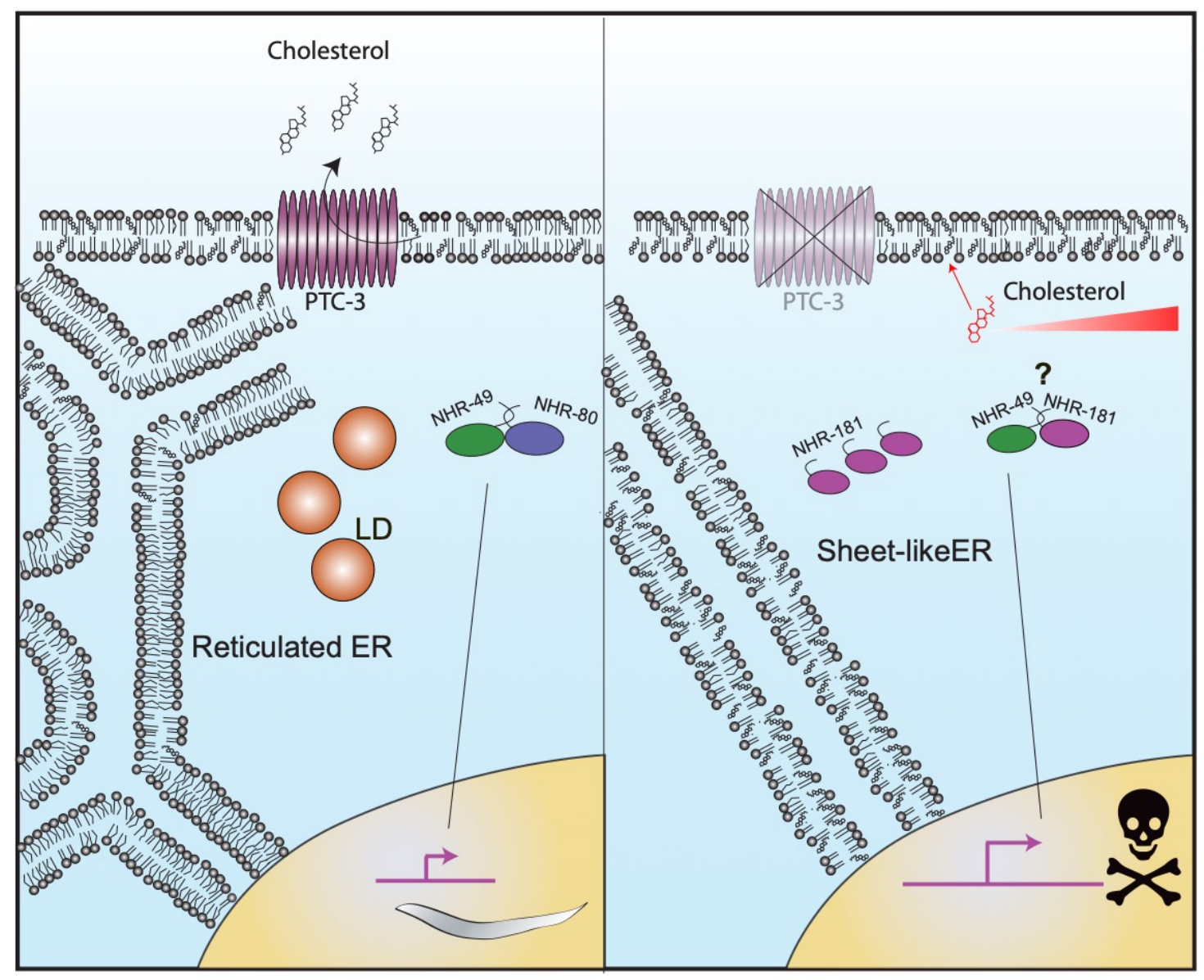


bioRxiv preprint doi: https://doi.org/10.1101/816256; this version posted October 23, 2019. The copyright holder for this preprint (which was not certified by peer review) is the author/funder, who has granted bioRxiv a license to display the preprint in perpetuity. It is made available under aCC-BY-ND 4.0 International license.

Figure S6

Mock

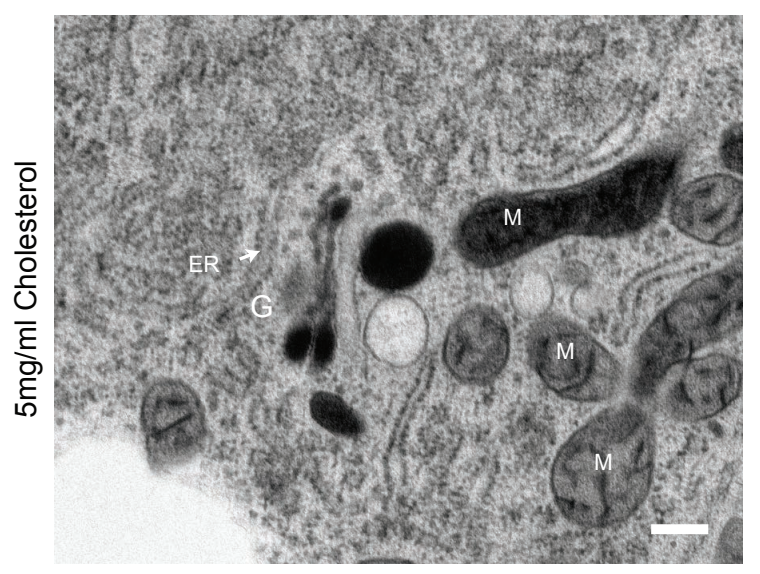

ptc-3(RNAi)

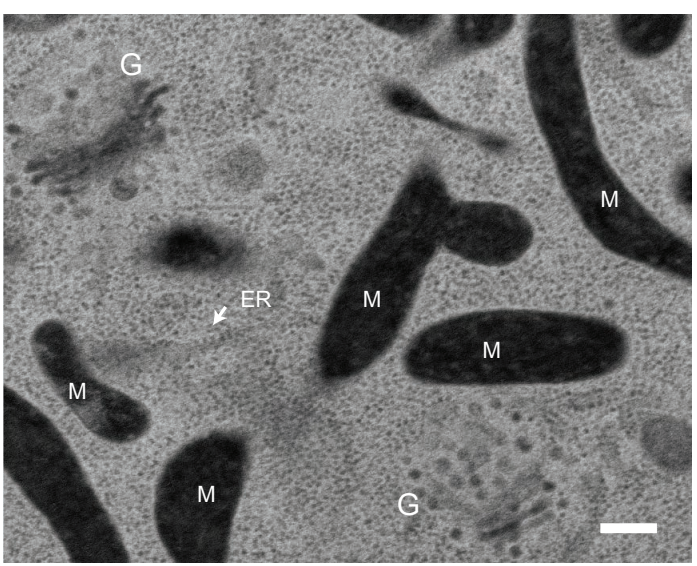


energetically favorable. The COPII coat can thus probably exert the force necessary to bend the ER membranes in the mutant.

However, the cholesterol accumulation may not only have a structural role in stiffening of membranes, together with MUFAs and saturated fatty acid acyl chains. It was shown previously that cholesterol can act as a hormone in C. elegans ${ }^{43-45}$. In support of this notion, we observed cuticular defects with intestine-specific knockdown on PTC-3, indicating that there are cell non-autonomous effects of ptc-3(RNAi). Given that PTC-3 is a cholesterol transporter, we speculate that the increased cholesterol levels are the causative of the cuticle defects. Moreover, cholesterol may also change the transcriptional program and reduce the expression of genes required for $F A$ desaturation and elongation. In fact, overexpression of the PPAR $\alpha$ homolog NHR-49 rescued the ptc-3(RNAi)-induced fat storage and developmental arrest phenotype. In mammalian cells, PPAR $\alpha$ requires PUFAs for activation ${ }^{35,46}$. We hypothesize that reduced PUFA levels downregulate NHR-49 activity, which could be compensated by the overexpression of NHR-49. Alternatively, but not mutually exclusive, the interaction between NHR-49 and NHR-80, which are jointly controlling FA elongation and desaturation ${ }^{36}$, would be disrupted by high cholesterol levels and NHR-49 would instead team up with NHR-181. This complex could then negatively regulate FAT and ELO gene expression when cholesterol levels increase in the cell. A circumstantial argument that put weight on this latter possibility is that both NHR-80 and NHR-181 are homologs of mammalian HNF4 proteins. Thus, it is tempting to speculate that the exchange of one HNF4 like molecule for another would shift the activity of NHR-49 from promoting elongation and desaturation to repressing these processes. Interestingly, a C-terminal truncation in Ptch1 in adult mice led to a reduction of white fat tissue and PPAR $\gamma$ levels, suggesting that the SMO-independent pathway we uncovered might be conserved in mammals ${ }^{47}$.

NHR-49 and NHR-181 appear to be specific downstream effectors of PTC-3 activity levels, as down regulating NHR-8 did not improve PTC-3-dependent phenotypes. Likewise, SREB, which is a major responder to alteration in cellular cholesterol levels and which has been shown to regulate the expression of fatty acid elongases and desaturases in mammalian cells ${ }^{48}$. However, knockdown of PTC-3 did not affect the nuclear localization of the $C$. elegans SREB homolog SBP-1 (Fig. S5C). However, NHR-49 activity clearly is affected by increased cholesterol levels since 
overexpression of its target and activator FAT-7 ${ }^{35}$ partially rescued ptc-3(RNAi) phenotypes.

We used $C$. elegans to reveal potential ancestral functions of PTCH family proteins because it lacks smoothened and other canonical hedgehog signaling pathway components, which have been lost during evolution ${ }^{22,23}$. In fact, it has been proposed that PTCH and related proteins such NPC1 and dispatched evolved separately from smoothened ${ }^{49}$. PTCH belongs to the family of RND transporters, which are already present in bacteria. For most bacterial RNDs the substrates are unknown. However, the family most related to PTCH transports hopanoids, which are structural and functional analogs of sterols ${ }^{49}$. Next to PTC-3, C. elegans encodes another PTCH protein PTC-1 and 18 PTCH-related proteins (PTRs), presumably all RND transporters. It is tempting to speculate that these PTCs and PTRs are transporting small molecules, presumably sterols, and thereby contributing to cellular homeostasis and potential intra- and intercellular communication.

Our data strongly implicate cellular cholesterol levels, membrane composition and nuclear hormone receptors such as PPAR $\alpha$ and HNF4 in non-canonical Hh signaling pathways. They also provide a framework on how to distinguish between SMO-dependent and -independent functions in mammals. Our results might in particularly important for the understanding of diseases such as multiple myeloma in which canonical and non-canonical Hh signaling have been implicated ${ }^{50,51}$. 


\section{Materials and methods}

\section{General methods and strains}

C. elegans was cultured and maintained as described previously ${ }^{52}$ at $20^{\circ} \mathrm{C}$ unless it was specified different. RNAi was carried out using sequenced and confirmed clones from the Ahringer library, as mock nontargeting dsRNA from the Ahringer library clone Y95B8A_84.g was used ${ }^{53}$. For low cholesterol conditions cholesterol was omitted and agar replaced by agarose. RNAi feeding experiments were performed for 3 days starting from L1 larvae. When adult ptc-3(RNAi) were needed worms were grown with RNAi mock bacteria until L2 stage and then transferred to ptc-3(RNAi) plates for 2 days. For developmental and survival assays eggs from 1-day adult worms were hatched in $\mathrm{M} 9$ buffer $\left(3 \mathrm{~g} \mathrm{KH}_{2} \mathrm{PO}_{4}, 6 \mathrm{~g} \mathrm{Na}_{2} \mathrm{HPO}_{4}, 5 \mathrm{~g} \mathrm{NaCl}, 1 \mathrm{ml} 1 \mathrm{M} \mathrm{MgSO}\right.$, $\mathrm{H}_{2} \mathrm{O}$ to $1 \mathrm{I}$; sterilized by autoclaving) overnight without bleaching. L1s were transferred to RNAi plates and grown at $20^{\circ} \mathrm{C}$. Survival and developmental stage were assessed after 72 hr. For double RNAi experiments ptc-3(RNAi) was diluted 1:1 with the second RNAi or mock expressing bacteria. nhr-49(nr2041) [ges-1p::3xHA::nhr-49(cDNA)::unc-54 3'UTR + myo-3p::mCherry::unc-54 3'UTR] and for gut specific RNAi kbls7 [nhx$2 p:: r d e-1+$ rol-6(su1006)] was used , sbp-1(ep79)

[sbp-1::GFP.:SBP-1; rol-6(su1006)], were obtained from the Caenorhabditis Genetics Center (CGC). nhr-8(hd117) mutant and nhr-8::GFP over expressing strains were described previously ${ }^{39}$. For the cholesterol sensor strains generation the PFO-derived D4 domain mutants YDA (D434W Y415A A463W) and YQDA (D434W Y415A A463W Q433W) fused to a mCherry N-terminal tag were cloned using the NEBuilder HiFi DNA Assembly Cloning Kit (NEB \#E5520) and introduced into pBlueScriptll with a VHA-6 promoter and tub terminator using the primers pvha6_fwd gacggtatcgataagcttgatatcggtatactatttattactcgatactttg, pvha6_rev cacgcttgccatttttatgggttttggtagg, for the promoter amplification, for the sensor amplification D4H_fwd aaacccataaaaaatggcaagcgtgagcaag, D4H_rev ttttgcatttatcttaattgtaagtaatactagatccagggtataaag were used, and tubter_fwd acttacaattaagataaatgcaaaatcctttcaag, tubter_rev actagtggatccccoggetgcaggtgagactttttcttggc for tubulin terminator. The plasmid was microinjected at a concentration of $50 \mathrm{ng} / \mu \mathrm{l}$ into both arms of the syncytial gonads of N2 worms. SUR-5::GFP at $10 \mathrm{ng} / \mu \mathrm{l}$ concentration was co-injected as transformation marker and $40 \mathrm{ng} / \mu \mathrm{l}$ of lambda DNA as carrier was used. Animals containing the 
cholesterol sensors were grown at $25^{\circ} \mathrm{C}$ and fed with OP50 RNAi-competent bacteria 54. The ptc-3::gfp reporter $\mathrm{pCH} 115.1$ was constructed by inserting a gfp cassette into the same site within the ptc-3 locus as described in ${ }^{18}$ in the fosmid WRM064cC06, following the recombineering protocol described in ${ }^{55}$. The gfp cassette was PCRamplified by using pBALU1 vector and primers $\mathrm{CH} 428$ gaaaaagagatttggcctactgcagtcgaggaaacccacaaatggcgactatgagtaaaggagaagaacttttca and

$\mathrm{CH} 429$ gccgaaaaactcgaacttacatttgaaacattgctcggcacactttgacttttgtatagttcatccatgcca, and the galK module was excised after its insertion to the fosmid. As co-injection marker pMF435: Ppgp-1::mCherry::unc-54 3'UTR was used ${ }^{56}$.

\section{Microscopy}

Live worms were immobilized with $50 \mathrm{mM}$ levamisole in $\mathrm{M} 9$ and mounted on a slide with $2 \%$ agarose. The worms were imaged with a Zeiss Axioplan 2 microscope equipped with a Zeiss Axio Cam MRm camera (Carl Zeiss, Aalen Oberkochen, Germany) and the objectives EC Plan-Neofluar 10x/0.3, EC Plan-Neofluar 20x/0.50, EC Plan-Neofluar 40/1.30. All images were adjusted to the same parameters with OMERO.web 5.3.4-ice36-b69. Images of D4H cholesterol sensors, Filipin III staining and TRAM::GFP were obtained on a Zeiss LSM 880 microscope with Airyscan with Plan-Apochromat 63x/1.4 Oil DIC M2. The fast mode was used, and images were processed using the Zen Black software.

\section{Coherent Anti-Stokes Raman Spectroscopy}

Worms were mounted on a slide with $2 \%$ agarose with $20 \mathrm{mM}$ levamisol. A Leica TCS SP8 system with a CARS laser picoEmerald. The lasers were beam to $816.4 \mathrm{~nm}$ while keeping the Stokes beam constant at $1,064.6 \mathrm{~nm}$. The scan speed was set to $400 \mathrm{~Hz}$. A z-stack per worm was imaged along the intestine and 19 animals from 3 experiments were collected per condition. The number of lipid droplets in each stack was assessed with the Fiji plug-in Lipid Droplet Counter. The data was analyzed with a one tail ANOVA followed by Dunnett's multiple comparisons test in Prism 7.

\section{TEM and FIB SEM}

For transmission electron microscopy (TEM) and focused-ion beam scanning electron microscopy (FIB-SEM), worms were frozen as follows. C. elegans animals were 
picked with a worm pick from agar plate and transferred to a droplet of M9 medium on a $100 \mu \mathrm{m}$ cavity of a $3 \mathrm{~mm}$ aluminium specimen carrier (Engineering office $\mathrm{M}$. Wohlwend $\mathrm{GmbH}$, Sennwald, Switzerland). 5 - 10 worms were added to the droplet and the excess M9 medium was sucked off with dental filter tips. A flat aluminium specimen carrier was dipped in 1-hexadecene and added on top. Immediately, the specimen carrier sandwich was transferred to the middle plate of an HPM 100 highpressure freezer (Leica Microsystems, Vienna, Austria) and frozen immediately without using ethanol as synchronizing medium.

Freeze-substitution was carried out in water-free acetone containing $1 \% \mathrm{OsO}_{4}$ for $8 \mathrm{hr}$ at $-90^{\circ} \mathrm{C}, 7 \mathrm{hr}$ at $-60^{\circ} \mathrm{C}, 5 \mathrm{hr}$ at $-30^{\circ} \mathrm{C}, 1 \mathrm{hr}$ at $0^{\circ} \mathrm{C}$, with transition gradients of $30^{\circ} \mathrm{C} / \mathrm{hr}$, followed by $30 \mathrm{~min}$ incubation at RT. Samples were rinsed twice with acetone water-free, block-stained with $1 \%$ uranyl acetate in acetone (stock solution: $20 \%$ in $\mathrm{MeOH}$ ) for $1 \mathrm{hr}$ at $4^{\circ} \mathrm{C}$, rinsed twice with water-free acetone and embedded in Epon/Araldite (Merck, Darmstadt, Germany): 66\% in acetone overnight, $100 \%$ for $1 \mathrm{hr}$ at RT and polymerized at $60^{\circ} \mathrm{C}$ for $20 \mathrm{hr}$. Ultrathin sections $(50 \mathrm{~nm})$ were post-stained with Reynolds lead citrate and imaged in a Talos 120 transmission electron microscope at $120 \mathrm{kV}$ acceleration voltage equipped with a bottom mounted Ceta camera using the Maps software (Thermo Fisher Scientific, Eindhoven, The Netherlands).

For Focused ion beam scanning electron tomography, a trimmed Epon/Araldite block containing a single $C$. elegans was mounted on a regular SEM stub using conductive carbon and coated with $10 \mathrm{~nm}$ of carbon by electron beam evaporation to render the sample conductive. Ion milling and image acquisition was performed simultaneously in an Auriga 40 Crossbeam system (Zeiss, Oberkochen, Germany) using the FIBICS Nanopatterning engine (Fibics Inc., Ottawa, Canada). A large trench was milled at a current of $16 \mathrm{nA}$ and $30 \mathrm{kV}$, followed by fine milling at $240 \mathrm{pA}$ and 30 $\mathrm{kV}$ during image acquisition with an advance of $5 \mathrm{~nm}$ per image. Prior to starting the fine milling and imaging, a protective Platinum layer of approximately $300 \mathrm{~nm}$ was applied on top of the surface of the area of interest using the single gas injection system at the FIB-SEM. SEM images were acquired at $1.9 \mathrm{kV}$ (30 $\mu \mathrm{m}$ aperture) using an in-lens energy selective backscattered electron detector (ESB) with a grid voltage of $550 \mathrm{~V}$, and a dwell time of $1 \mu \mathrm{s}$ and a line averaging of 130 lines. The pixel size was set to $5 \mathrm{~nm}$ and tilt-corrected to obtain isotropic voxels. The final image stack was registered and cropped to the area of interest using the Fiji image-processing package 
[https://imagej.net/TrakEM2]. FIB-SEM images were processed with iLastik $^{57}$ and pixel classification was done. The classifier was trained to separate different object classes, ER, cytoplasm and other organelles. The training was done individually for each dataset. A 3D reconstruction was later handled with IMARIS 9.2.

\section{Lipidomic analysis}

Worms were cultured in liquid media as described previously ${ }^{58}$. Feeding bacteria were prepared by growing RNAi bacteria to an $\mathrm{OD}_{600}$ of 0.6 in LB-Amp medium and then inducing dsRNA expression with $1 \mathrm{mM}$ IPTG for $24 \mathrm{hr}$. Bacteria were harvested, resuspended to $\mathrm{OD}_{600} 400$. Synchronized populations of worms were grown from L1 larvae to L2 stage in mock bacteria and then transferred into RNAi bacteria until they reach early adulthood. Young adults were collected and washed once in $\mathrm{ddH}_{2} \mathrm{O} .8,000$ young adults were used for glycerophospholipid and sphingolipid analysis while sterol analysis was done from 40,000 young adults. Pellets were frozen and stored at $-80^{\circ} \mathrm{C}$ until extraction. Lysis was performed on a Cryolysis machine (Precellys 24, lysis \& homogenization machine (Bertin Technologies)) at $4^{\circ} \mathrm{C}$ using $100 \mu 1.4 \mathrm{~mm}$ zirconium oxide beads in $800 \mu \mathrm{MS}-\mathrm{H}_{2} \mathrm{O}$ with three cycles of $45 \mathrm{sec}$ bursts at 6,200 rpm followed by $45 \mathrm{sec}$ interruptions. Lysates were eluted into glass tubes with lipid standards (glycerophospholipid and sphingolipid standards: di-lauryl phosphatidylcholine, dilauryl phosphatidylethanolamine, di-lauryl phosphatidylinositol, di-lauryl phosphatidylserine, tetra-lauryl cardiolipin, C17 ceramide, C12 sphingomyelin, C8 glucosylceramide, all from Avanti Polar Lipids; sterol standard: ergosterol from Fluka) and beads were washed and eluted again with $200 \mu \mathrm{MS}-\mathrm{H}_{2} \mathrm{O}$. Lipids were extracted with chloroform and methanol according to Bligh and Dyer ${ }^{59}$ following a published protocol ${ }^{60}$. Briefly, $3.6 \mathrm{ml}$ organic solvent $\left(\mathrm{CHCl}_{3} / \mathrm{MeOH}=1: 2\right.$, v:v) were added to the $1 \mathrm{ml}$ aqueous lysate, mixed and centrifuged to clear extract from worm debris. Extracts were transferred to new glass tubes and phase separation was induced by addition of $0.5 \mathrm{~mL} \mathrm{MS}-\mathrm{H}_{2} \mathrm{O}$ and $0.5 \mathrm{ml} \mathrm{CHCl}_{3}$. Samples were centrifuged, and the organic phase was collected. For sterol analysis total lipid extract was dried directly in a centrivap. To separate sterols from other lipids solid phase extraction on a Chromabond $\mathbb{S i O H}$ column (Macherey-Nagel, Germany) was performed. Columns were washed two times with $1 \mathrm{ml} \mathrm{CHCl}$. Total lipid extract from 40,000 worms was resuspended in 250 $\mu \mathrm{CHCl}_{3}$ by vortexing and sonication. The extract was then applied to the column and eluted with two times $650 \mu \mathrm{CHCl}_{3}$. The flow-through and $\mathrm{CHCl}_{3}$ elutions were 
combined, dried and used for sterol analysis by GC-MS. In the case of glycerophospholipid and sphingolipid analysis, total lipid extract was split in two and dried. One aliquot was used without further treatments for glycerophospholipid analysis and inorganic phosphate determination while the other underwent methylamine treatment and desalting via butanol extraction ${ }^{61}$.

Glycerophospholipid and sphingolipid analysis was performed following a worm adapted version of a previously published method ${ }^{62}$. LC-MS or HPLC grade solvents were used and the samples were pipetted in a 96 well plate (final volume $=100 \mu \mathrm{l}$ ). Positive mode solvent: $\mathrm{CHCl}_{3} / \mathrm{MeOH} / \mathrm{H}_{2} \mathrm{O}(2: 7: 1 \mathrm{v} / \mathrm{v})+5 \mathrm{mM} \mathrm{NH}_{4} \mathrm{Ac}$. Negative mode solvent: $\mathrm{CHCl}_{3} / \mathrm{MeOH}(1: 2 \mathrm{v} / \mathrm{v})+5 \mathrm{mM} \mathrm{NH} \mathrm{N}_{4} \mathrm{Ac}$. The glycerophospholipid and sphingolipid aliquots were resuspended in $250 \mu \mathrm{CHCl}_{3} / \mathrm{MeOH}(1: 1 \mathrm{v} / \mathrm{v})$ and sonicated for $5 \mathrm{~min}$. The glycerophospholipids were diluted 1:10 in negative and positive mode solvents and the sphingolipids were diluted 1:5 in positive mode solvent and infused onto the mass spectrometer. Tandem mass spectrometry for the identification and quantification of glycerophospholipid and sphingolipid molecular species was performed using multiple reaction monitoring (MRM) with a TSQ Vantage Triple Stage Quadrupole Mass Spectrometer (Thermo Fisher Scientific, Bremen, Germany) equipped with a robotic nanoflow ion source, Nanomate HD (Advion Biosciences, Ithaca, NY). The collision energy was optimized for each lipid class. Each biological replicate was read in two technical replicates each comprising three measurements for each transition. Lipid concentrations were calculated relative to the corresponding internal standards and then normalized to the total phosphate content of each total lipid extract. Sterol analysis was done as previously described ${ }^{61}$.

\section{Nile Red Staining}

Nile Red staining was performed as described previously ${ }^{63}$. Worms were washed with $1 \mathrm{ml} \mathrm{M9}$ into a $1.5 \mathrm{ml}$ siliconized microfuge tube. Worms were allowed to sink by gravity on ice and were washed with M9. Approximately $30 \mu \mathrm{l}$ of M9 and worms at the bottom of the tube were left. $0.2 \mathrm{ml}$ of $40 \%$ isopropanol was added and incubated for $3 \mathrm{~min}$ for fixation. The fixative was removed and $150 \mu \mathrm{l}$ of Nile Red solution (6 $\mu \mathrm{l}$ of Nile Red $0.5 \mathrm{mg} / \mathrm{ml}$ in acetone per $1 \mathrm{ml}$ of $40 \%$ isopropanol) was added to the worms for $30 \mathrm{~min}$ at $20^{\circ} \mathrm{C}$ with gentle rocking in the dark. Worms were washed once with $1 \mathrm{ml} \mathrm{M9}$ buffer and mounted on a $2 \%$ agarose pad for microscopy. Intensity analysis was performed 
using Fiji with at least 11 worms per condition from 3 different experiments. The data was analyzed with a one tail ANOVA followed by Dunnett's multiple comparisons test in Prism 7.

\section{TopFluor cholesterol staining}

The experiment was performed as described previously as ${ }^{5}$. PTC-3 CDS was amplified with the primers ptc-3TEF_F 5'actagtggatccccogggctgcaggATGAAGGTGCATTCGGAACAAC-3' ptc-3TEF_R 5'gacggtatcgataagettgatatcgTTACTTGTGCGCTGGCGATG-3' from cDNA and cloned into the yeast plasmid p426TEF. A point mutation (D697A) was introduced with the Q5® Site-Directed Mutagenesis Kit (E0554S NEB). Yeasts were cultured to an $\mathrm{OD}_{600}$ of 4 , washed with cold water and resuspend to $10 \mathrm{OD}_{600}$ in $50 \mathrm{mM}$ HEPES buffer $\mathrm{pH}$ 7.0. Yeasts were incubated protected from light with $5 \mu \mathrm{M}$ TopFluor ${ }^{\circledR}$ Cholesterol (810255, Avanti Polar Lipids) for $2 \mathrm{hr}$ at $20^{\circ} \mathrm{C}$. They were washed once with cold $\mathrm{ddH}_{2} \mathrm{O}$ and resuspend with HEPES buffer, after 20 min the yeast were spun down, and the supernatant was measured with filters $485 \mathrm{ex} 520 \mathrm{em}$ on a plate reader (DTX880, Multimode Detector, Beckman Coulter). The efflux was normalized to the initial fluorescence of the yeast. Worms were washed off a plate with M9 buffer and put on a shaker in M9 buffer with $5 \mathrm{mM}$ TopFluor ${ }^{\circledR}$ Cholesterol for $1 \mathrm{hr}$ at $20^{\circ} \mathrm{C}$. Worms were washed with M9 buffer once to remove the excess of Topfluor® Cholesterol and chase in M9 buffer was performed for $1 \mathrm{hr}$ before imaging.

\section{Immunofluorescence and PTC-3 antibody.}

Immunofluorescence of $C$. elegans was performed as described previously ${ }^{64}$, with slight modifications: Worms were blocked with PTB (1\% BSA, 1x PBS, $0.1 \%$ Tween20, $0.05 \% \mathrm{NaN}_{3}, 1 \mathrm{mM}$ EDTA) and secondary antibody was diluted in PTB. Peptide antibodies against $C$. elegans PTC-3 were generated in rabbits by Eurogentec using peptides SASHSSDDESSPAHK and EVRRGPELPKENGLG. Serum was used in a 1:100 dilution and Alexa Fluor 488-goat anti-rabbit IgG $(\mathrm{H}+\mathrm{L})$ (Invitrogen; A11034) 1:5,000. Worms were washed $2 x$ in $M 9$ and mounted with fluorescence protecting media (ProLong ${ }^{\mathrm{TM}}$ Glass Antifade Mountant Invitrogen P36984). Worms were imaged on a Zeiss LSM 880 microscope as described in the Microscopy section. 


\section{Filipin staining}

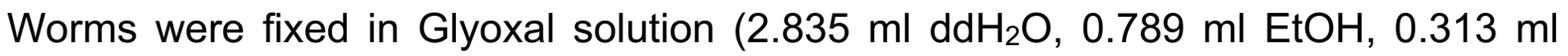
glyoxal (40\% stock solution from Sigma-Aldrich, \#128465) $0.03 \mathrm{ml}$ glacial acetic acid. $\mathrm{pH} 4.5$ ) for $30 \mathrm{~min}$ on ice, and for another $30 \mathrm{~min}$ at RT, followed by $30 \mathrm{~min}$ of quenching in $100 \mathrm{mM} \mathrm{NH}_{4} \mathrm{Cl}$ at $\mathrm{RT}$ and $\mathrm{O} / \mathrm{N}$ post quenching at $4^{\circ} \mathrm{C}{ }^{65}$. Worms were washed 2x 30 min with M9 and left in $50 \mu$ of M9 in which $50 \mu$ of Filipin III readymade solution (Sigma-Aldrich, SAE0087) was added for $1 \mathrm{hr}$ in the dark at RT. Worms were washed 2x in M9 and mounted with fluorescence protecting media (ProLong ${ }^{\mathrm{TM}}$ Glass Antifade Mountant Invitrogen P36984). Worms were imaged on a Zeiss LSM 880 microscope as described in the Microscopy section.

\section{Western Blot}

Worm Lysate from synchronous L3 worm cultures was prepared in Laemmli buffer with $6 \mathrm{M}$ urea with glass beads in a FastPrep machine (MP Biomedicals, Irvine, CA) for 2 x $30 \mathrm{sec}$. Samples were run on a 7.5\% SDS-PAGE before transfer onto nitrocellulose membranes (Amersham Protran; 10600003). Membranes were blocked in TBS containing 5\% milk for $1 \mathrm{hr}$ at RT. First antibody incubation was done O/N at $4^{\circ} \mathrm{C}$ and the secondary HRP-coupled antibodies goat anti-Mouse $\lg \mathrm{G}(\mathrm{H}+\mathrm{L})$ (ThermoFisher scientific; 31430; 1:10,000) or polyclonal HRP-conjugated goat-antirabbit IgG (ThermoFisher scientific; 31460; 1:10,000) for $1 \mathrm{hr}$ at RT. The blots were developed using WesternBright ECL HRP substrate (K-12045 Advansta) in a Fusion FX7 (Vilber Lourmat) image acquisition system.

\section{qRT-PCR}

RNA for qRT-PCR was extracted with TRIzol according to the manufacturer's instructions from synchronous worms $26 \mathrm{hr}$ after L1. The RNA was DNase digested and reverse transcribed using Maxima H Minus First Strand cDNA Synthesis Kit, with dsDNase (ThermoFischer Scientific). The resulting cDNA was diluted 1:10 for further analysis. The StepOne RT-PCR system combined with StepOne Software (Applied Biotechnologies) was used for analysis. The presented values are based on three biological replicates. Expression levels were normalized to cdc-42 Primer sequences: nhr41_F 5'- ACGTCGAGTCGTCCACATTT-3', nhr41_R 5'TCAGATCTCCCGAGCTCAAT-3', nhr181_F 5'-TGCGGAACAAAAAGCAGAGC-3', 
nhr181_R 5'-ATCTTTGTAGGTTACGTGACCC-3', cdc42_F 5'CCTCTATCGTATCCACAG-3', cdc42_R 5'-GGTCTTTGAGCAATGATG-3', nhr168_F 5'- GGGAACTGGCACCAATGAAG-3', nhr168_R 5'GTTGCGAGAGGTCAGGCACCG-3'. The data was analyzed with a two-way ANOVA followed by uncorrected Fisher's LSD test in Prism 7.

\section{Author contributions}

AS and CECC wrote the manuscript and designed the experiments. CECC performed the majority of the experiments. TH and HR performed the lipidomic analysis. AK generated the TEM and FIB-SEM data. HC and MF generated the PTC-3-GFP $C$. elegans strain. NF supervised the CARS experiments. AS, CECC, TH, HR and AK analyzed the data. All authors commented on the manuscript.

\section{Acknowledgements}

We thank J. Fürst, J. Stevens, J. Solinger, A. Stetak (Division of Molecular Neuroscience, University of Basel), I. Katic (FMI, Basel), the IMCF-Biozentrum and Microscopy Center of the University of Zürich for the technical support, and Y. Hauser (FMI, Basel) for helpful discussions. We thank J. Brewer and V. Solovyeva (University of Southern Denmark) for the help with the CARS experiments. S. Mango, T. Bürglin and M. Labouesse are acknowledged for critical reading of the manuscript. We thank A. Antebi for sharing strains. Some strains were provided by the CGC, which is funded by NIH Office of Research Infrastructure Programs (P40 OD010440). The project was supported by the Swiss National Science Foundation (CRSII3_141956) (AS), JSPS KAKENHI Grant Number 18K06246 (MF) and the University of Basel.

\section{References}

1. Zhang, Y. et al. Structural Basis for Cholesterol Transport-like Activity of the Hedgehog Receptor Patched. Cell 175, 1352-1364.e14 (2018).

2. Hahn, H. et al. Mutations of the human homolog of Drosophila patched in the nevoid basal cell carcinoma syndrome. Cell 85, 841-51 (1996).

3. Roberts, B., Casillas, C., Alfaro, A. C., Jägers, C. \& Roelink, H. Patched1 and Patched2 inhibit Smoothened non-cell autonomously. Elife 5, (2016).

4. Weiss, L. E., Milenkovic, L., Yoon, J., Stearns, T. \& Moerner, W. E. Motional 
dynamics of single Patched1 molecules in cilia are controlled by Hedgehog and cholesterol. Proc. Natl. Acad. Sci. U. S. A. 116, 5550-5557 (2019).

5. Bidet, M. et al. The hedgehog receptor patched is involved in cholesterol transport. PLoS One 6, e23834+ (2011).

6. Myers, B. R., Neahring, L., Zhang, Y., Roberts, K. J. \& Beachy, P. A. Rapid, direct activity assays for Smoothened reveal Hedgehog pathway regulation by membrane cholesterol and extracellular sodium. Proc. Natl. Acad. Sci. U. S. A. 114, E11141--E11150 (2017).

7. Huang, P. et al. Cellular Cholesterol Directly Activates Smoothened in Hedgehog Signaling. Cell 166, (2016).

8. Qi, X., Schmiege, P., Coutavas, E. \& Li, X. Two Patched molecules engage distinct sites on Hedgehog yielding a signaling-competent complex. Science (80-. ). eaas8843+ (2018). doi:10.1126/science.aas8843

9. Qi, X., Schmiege, P., Coutavas, E., Wang, J. \& Li, X. Structures of human Patched and its complex with native palmitoylated sonic hedgehog. Nature 560, 128-132 (2018).

10. Gong, X. et al. Structural basis for the recognition of Sonic Hedgehog by human Patched1. Science 361, eaas8935+ (2018).

11. Gu, D. \& Xie, J. Non-Canonical Hh Signaling in Cancer-Current Understanding and Future Directions. Cancers (Basel). 7, 1684-98 (2015).

12. Brennan, D., Chen, X., Cheng, L., Mahoney, M. \& Riobo, N. A. Noncanonical Hedgehog signaling. Vitam. Horm. 88, 55-72 (2012).

13. Chang, H., Li, Q., Moraes, R. C., Lewis, M. T. \& Hamel, P. A. Activation of Erk by sonic hedgehog independent of canonical hedgehog signalling. Int. J. Biochem. Cell Biol. 42, 1462-1471 (2010).

14. Regan, J. L. et al. Non-Canonical Hedgehog Signaling Is a Positive Regulator of the WNT Pathway and Is Required for the Survival of Colon Cancer Stem Cells. Cell Rep. 21, 2813-2828 (2017).

15. Bürglin, T. R. \& Kuwabara, P. E. Homologs of the Hh signalling network in C. elegans. WormBook 1-14 (2006).

16. Hao, L., Johnsen, R., Lauter, G., Baillie, D. \& Bürglin, T. R. Comprehensive analysis of gene expression patterns of hedgehog-related genes. BMC Genomics 7, 280 (2006).

17. Zugasti, O., Rajan, J. \& Kuwabara, P. E. The function and expansion of the 
Patched- and Hedgehog-related homologs in C. elegans. Genome Res. 15, 1402-1410 (2005).

18. Soloviev, A., Gallagher, J., Marnef, A. \& Kuwabara, P. E. C. elegans patched-3 is an essential gene implicated in osmoregulation and requiring an intact permease transporter domain. Dev. Biol. 351, 242-253 (2011).

19. Kuwabara, P. E., Lee, M. H., Schedl, T. \& Jefferis, G. S. X. E. A C. elegans patched gene, ptc-1, functions in germ-line cytokinesis. Genes Dev. 14, 19331944 (2000).

20. Cao, J. et al. Comprehensive single-cell transcriptional profiling of a multicellular organism. Science (80-. ). 357, 661-667 (2017).

21. Aspöck, G., Kagoshima, H., Niklaus, G. \& Bürglin, T. R. Caenorhabditis elegans has scores of hedgehog-related genes: sequence and expression analysis. Genome Res. 9, 909-23 (1999).

22. Bürglin, T. R. Evolution of hedgehog and hedgehog-related genes, their origin from Hog proteins in ancestral eukaryotes and discovery of a novel Hint motif. BMC Genomics 9, 127 (2008).

23. Bürglin, T. R. The Hedgehog protein family. Genome Biol. 9, 241 (2008).

24. Hodgkin, J. A. \& Brenner, S. Mutations causing transformation of sexual phenotype in the nematode Caenorhabditis elegans. Genetics 86, 275-87 (1977).

25. Mullaney, B. C. \& Ashrafi, K. C. elegans fat storage and metabolic regulation. Biochim. Biophys. Acta 1791, 474-478 (2009).

26. Espelt, M. V., Estevez, A. Y., Yin, X. \& Strange, K. Oscillatory Ca ${ }^{2+}$ Signaling in the Isolated Caenorhabditis elegans Intestine. J. Gen. Physiol. 126, 379-392 (2005).

27. Habacher, C. et al. Ribonuclease-Mediated Control of Body Fat. Dev. Cell 39, 359-369 (2016).

28. Hellerer, T. et al. Monitoring of lipid storage in Caenorhabditis elegans using coherent anti-Stokes Raman scattering (CARS) microscopy. Proc. Natl. Acad. Sci. U. S. A. 104, 14658-63 (2007).

29. Xu, S. H. \& Nes, W. D. Biosynthesis of cholesterol in the yeast mutant erg6. Biochem. Biophys. Res. Commun. 155, 509-17 (1988).

30. Maekawa, M. Domain 4 (D4) of Perfringolysin $O$ to Visualize Cholesterol in Cellular Membranes-The Update. Sensors (Basel). 17, (2017). 
31. Liu, S.-L. et al. Orthogonal lipid sensors identify transbilayer asymmetry of plasma membrane cholesterol. Nat. Chem. Biol. 13, 268-274 (2017).

32. Marek, M., Vincenzetti, V. \& Martin, S. G. Sterol flow between the plasma membrane and the endosome is regulated by the LAM family protein Ltc1. doi:10.1101/720383

33. Hieb, W. F. \& Rothstein, M. Sterol requirement for reproduction of a free-living nematode. Science (80-. ). 160, 778-780 (1968).

34. Poteryaev, D., Squirrell, J. M., Campbell, J. M., White, J. G. \& Spang, A. Involvement of the Actin Cytoskeleton and Homotypic Membrane Fusion in ER Dynamics in Caenorhabditis elegans. Mol. Biol. Cell 16, 2139-2153 (2005).

35. Ma, D. K. et al. Acyl-CoA Dehydrogenase Drives Heat Adaptation by Sequestering Fatty Acids. Cell 161, 1152-1163 (2015).

36. Pathare, P. P., Lin, A., Bornfeldt, K. E., Taubert, S. \& Van Gilst, M. R. Coordinate regulation of lipid metabolism by novel nuclear receptor partnerships. PLOS Genet. 8, e1002645+ (2012).

37. Van Gilst, M. R., Hadjivassiliou, H., Jolly, A. \& Yamamoto, K. R. Nuclear hormone receptor NHR-49 controls fat consumption and fatty acid composition in C. elegans. PLoS Biol. 3, e53 (2005).

38. Ashrafi, K. et al. Genome-wide RNAi analysis of Caenorhabditis elegans fat regulatory genes. Nature 421, 268-272 (2003).

39. Magner, D. B. et al. The NHR-8 nuclear receptor regulates cholesterol and bile acid homeostasis in C. elegans. Cell Metab. 18, 212-224 (2013).

40. Hendriks, G.-J. J., Gaidatzis, D., Aeschimann, F. \& Großhans, H. Extensive oscillatory gene expression during C. elegans larval development. Mol. Cell 53, 380-392 (2014).

41. Taipale, J., Cooper, M. K., Maiti, T. \& Beachy, P. A. Patched acts catalytically to suppress the activity of Smoothened. Nature 418, 892-897 (2002).

42. Matsuoka, K. et al. COPII-coated vesicle formation reconstituted with purified coat proteins and chemically defined liposomes. Cell 93, 263-275 (1998).

43. Hannich, J. T. et al. Methylation of the Sterol Nucleus by STRM-1 Regulates Dauer Larva Formation in Caenorhabditis elegans. Dev. Cell 16, 833-843 (2009).

44. Matyash, V. et al. Sterol-Derived Hormone(s) Controls Entry into Diapause in Caenorhabditis elegans by Consecutive Activation of DAF-12 and DAF-16. 
PLoS Biol. 2, e280 (2004).

45. Kurzchalia, T. V. \& Ward, S. Why do worms need cholesterol? Nat. Cell Biol. 5, 684-688 (2003).

46. Dhe-Paganon, S., Duda, K., Iwamoto, M., Chi, Y.-I. \& Shoelson, S. E. Crystal Structure of the HNF4a Ligand Binding Domain in Complex with Endogenous Fatty Acid Ligand. J. Biol. Chem. 277, 37973-37976 (2002).

47. Li, Z. et al. Reduced white fat mass in adult mice bearing a truncated Patched 1. Int. J. Biol. Sci. 4, 29-36 (2008).

48. Walker, A. K. et al. A conserved SREBP-1/phosphatidylcholine feedback circuit regulates lipogenesis in metazoans. Cell 147, 840-52 (2011).

49. Hausmann, G., von Mering, C. \& Basler, K. The Hedgehog Signaling Pathway: Where Did It Come From? PLoS Biol. 7, e1000146 (2009).

50. Agarwal, J. R. et al. Activation of Liver X Receptors Inhibits Hedgehog Signaling, Clonogenic Growth, and Self-Renewal in Multiple Myeloma. Mol. Cancer Ther. 13, 1873-1881 (2014).

51. Blotta, S. et al. Canonical and noncanonical Hedgehog pathway in the pathogenesis of multiple myeloma. Blood 120, 5002-5013 (2012).

52. Brenner, S. The genetics of Caenorhabditis elegans. Genetics 77, 71-94 (1974).

53. Kamath, R. S. et al. Systematic functional analysis of the Caenorhabditis elegans genome using RNAi. Nature 421, 231-237 (2003).

54. Xiao, R. et al. RNAi Interrogation of Dietary Modulation of Development, Metabolism, Behavior, and Aging in C. elegans. Cell Rep. 11, 1123-1133 (2015).

55. Tursun, B., Cochella, L., Carrera, I. \& Hobert, O. A Toolkit and Robust Pipeline for the Generation of Fosmid-Based Reporter Genes in C. elegans. PLoS One 4, e4625 (2009).

56. Fukuyama, M., Kontani, K., Katada, T. \& Rougvie, A. E. The C. elegans Hypodermis Couples Progenitor Cell Quiescence to the Dietary State. Curr. Biol. 25, 1241-1248 (2015).

57. Sommer, C., Straehle, C., Koethe, U. \& Hamprecht, F. A. Ilastik: Interactive learning and segmentation toolkit. in Biomedical Imaging: From Nano to Macro, 2011 IEEE International Symposium on 230-233 (2011).

58. Stiernagle, T. Maintenance of C. elegans. WormBook (2006). 
doi:10.1895/wormbook.1.101.1

59. BLIGH, E. G. \& DYER, W. J. A rapid method of total lipid extraction and purification. Can. J. Biochem. Physiol. 37, 911-917 (1959).

60. Schwudke, D. et al. Top-Down Lipidomic Screens by Multivariate Analysis of High-Resolution Survey Mass Spectra. Anal. Chem. 79, 4083-4093 (2007).

61. Guri, Y. et al. mTORC2 Promotes Tumorigenesis via Lipid Synthesis. Cancer Cell 32, 807-823.e12 (2017).

62. da Silveira Dos Santos, A. X. et al. Systematic lipidomic analysis of yeast protein kinase and phosphatase mutants reveals novel insights into regulation of lipid homeostasis. Mol. Biol. Cell 25, 3234-46 (2014).

63. Pino, E. C., Webster, C. M., Carr, C. E. \& Soukas, A. A. Biochemical and high throughput microscopic assessment of fat mass in Caenorhabditis elegans. $J$. Vis. Exp. (2013). doi:10.3791/50180

64. Ackema, K. B., Sauder, U., Solinger, J. A. \& Spang, A. The ArfGEF GBF-1 Is Required for ER Structure, Secretion and Endocytic Transport in C. elegans. PLoS One 8, e67076 (2013).

65. Richter, K. N. et al. Glyoxal as an alternative fixative to formaldehyde in immunostaining and super-resolution microscopy. EMBO J. 37, 139-159 (2018). 


\section{Figure legends}

Figure 1. Loss of PTC-3 causes developmental and fat storage defects. (A) Light microscopy images of adult N2 worms grown from L2 larva on mock or ptc-3(RNAi) bacteria. ptc-3(RNAi) animals are smaller, paler and show cuticle defects. The arrow points to a cuticular defect. Scale bars upper panel $20 \mu \mathrm{m}$, lower panels $100 \mu \mathrm{m}$. (B) Immunofluorescence of isolated intestine and gonad from wild-type worms. Schematic representation of the intestine and the proximal gonad. PTC-3 is present at the intestinal apical membrane. Scale bar $10 \mu \mathrm{m}$. (C) PTC-3 has cell autonomous and non-cell autonomous functions. Light microscopy images of adult gut specific RNAi worms (RNA ${ }^{\text {gut }}$ ) grown from L1 larva on mock or ptc-3(RNAi) bacteria. ptc-3(RNAigut) animals show intestinal and vulval defects. Scale bars $100 \mu \mathrm{m}$. (D) Lipid content is reduced in ptc-3(RNAi) animals. Nile Red staining of mock and ptc-3(RNAi) treated worms. Scale bars $20 \mu \mathrm{m}$. (E) Quantification of Nile Red staining shown in (D). Error bars are SEM. ${ }^{* * *} p<0.0001$. (F) CARS microscopy reveals reduction of lipid levels in live ptc-3(RNAi) animals. Scale bars $10 \mu \mathrm{m}$. The intestine is outlined by green dashed lines. Arrows point to the intestine. (G) Quantification of CARS signal. Error bars are SEM. ${ }^{* * *} p<0.0001$.

Figure 2. PTC-3 is a cholesterol transporter. (A) S. cerevisiae expressing PTC-3 or PTC-3 $3^{\text {D697A }}$ were incubated for 2 hr with $2.5 \mathrm{mM}$ TopFluor ${ }^{\circledR}$ Cholesterol, washed, resuspended in cholesterol free buffer, and after $20 \mathrm{~min}$, fluorescence intensity of the supernatant was measured. PTC-3 expression induced cholesterol efflux from yeast, which was abolished by the D697A mutation. Error bars are SD. ${ }^{* * *} p<0.0001$ (B) N2 worms fed with mock or ptc-3(RNAi) were incubated with $2.5 \mathrm{mM}$ TopFluor $\circledast$ Cholesterol for $1 \mathrm{hr}$. After $1 \mathrm{hr}$ chase, animals were imaged. Scale bar $10 \mu \mathrm{m}$. (C) Cholesterol accumulates in ptc-3(RNAi) animals. Quantification of sterols by MS. Error bars are SEM. ${ }^{* * *} p<0.0001$. (D) Cholesterol identification by Filipin or mutagenized YDA or YQDA D4::mCherry cholesterol sensor in the worm gut in mock or ptc-3(RNAi) treated animals. ptc-3 (RNAi) induces membranal cholesterol accumulation in the intestinal apical membrane. Scale bars $10 \mu \mathrm{m}$. (E) Quantification of apical membrane enrichment over cytoplasm of the cholesterol sensors YDA or YQDA D4::mCherry. *** $p<0.0001$. 
Figure 3. Low dietary cholesterol rescues ptc-3(RNAi) induced phenotypes. Worms feed from L1 larva under standard cholesterol conditions (5 mg/l) or low cholesterol conditions (no added cholesterol) for 3 days. (A) Representative light microscopy images of mock- or ptc-3(RNAi)-treated worms on low cholesterol plates Scale bars $20 \mu \mathrm{m}$. (B) Quantification of number of ptc-3(RNAi)-treated worms that reached adulthood in the absence of cholesterol in the growth medium. Error bars are SEM. ${ }^{* * *} p<0.0001$. (C) Nile Red staining of lipid droplets in C. elegans mock- and ptc3(RNAi)-treated animals on normal or low cholesterol conditions. In low cholesterol conditions, lipid droplet levels are restored in ptc-3(RNAi) animals. Scale bars $20 \mu \mathrm{m}$. (D) Quantification of Nile Red staining of data shown in (C). The normal cholesterol data are the same as depicted in Fig. 1E.

Figure 4. ptc-3(RNAi) reduces LD in the gut and induces changes in the ER structure. (A) Transmission electron microscopy (TEM) of mock and ptc-3(RNAi) treated animals reveal a reduction of reticulate ER structures and LD in ptc-3(RNAi) animals. This phenotype is rescued by omission of cholesterol in the medium. ER: Endoplasmic reticulum, LD: lipid droplet, M: mitochondria, G: Golgi. Scale bars 200 $\mathrm{nm}$. (B) Reconstitution of ER membranes from FIB-SEM images of mock and ptc$3(R N A i)$ treated animals using machine learning. ptc-3(RNAi) induces sheet-like ER structures.

Figure 5. ptc-3(RNAi) decreases phospholipid FA saturation and elongation. (A) Increasing the growth temperature from $20^{\circ} \mathrm{C}$ to $25^{\circ} \mathrm{C}$ improves the development of ptc-3(RNAi) animals. Representative bright field pictures of worms on growth plates. (B) Quantification of ptc-3(RNAi) survivors at both temperatures. Error bars are SEM. ${ }^{*} p<0.05$. (C) Lipidomics on mock or RNAi treated worms. ptc-3(RNAi) worms showed no difference in lipid head group distribution. Error bars are SEM. (D) Lipidomics revealed differences in lipid acyl chain composition upon ptc-3(RNAi). There is a shift from PUFAs to saturated FA and MUFAs. Error bars are SEM.

Figure 6. PTC-3 influences NHR function in a cholesterol-dependent manner. (A) Overexpression of NHR-49 or FAT-7 partially rescues ptc-3(RNAi) defects. Representative DIC images of worms. Scale bars $100 \mu \mathrm{m}$. (B) Quantification of survival rate upon overexpression of FAT-7, NHR-49 or NHR-80 in ptc-3(RNAi) 
animals. Error bars are SD. ${ }^{* *} p<0.0001$. (C) Over expression of NHR-49 partially restores fat accumulation in ptc-3(RNAi) animals. Nile Red staining. Scale bars $20 \mu \mathrm{m}$. (D) Quantification of data shown in (C). (E) Schematic representation of ribosomal footprints of mRNA during $C$. elegans larval development. Oscillatory changes in mRNA levels during developmental time. The timing, amplitude and whether a gene is oscillating is gene specific. (F) PTC-3, but not NHR-49 or NHR-80, expression oscillates during development. Data plotted from ${ }^{40}$. (G) Ribosomal footprint oscillations of NHR-181, NHR-168 and NHR-41 are similar to PTC-3 (data from ${ }^{40}$ ). (H) NHR-181 expression is modulated dependent on cholesterol levels. qRT-PCR analysis of NHR-41, NHR-168 and NHR-181 in the presence or absence of cholesterol in the growth medium. Error bars are SEM. ${ }^{* *} p<0.001{ }^{*} p<0.05$. (I) Genetic interaction between PTC-3 and NHR-181. Knockdown of NHR-181 rescues ptc-3(RNAi) lethality. (J) $n h r-181$ (RNAi) partially restores fat accumulation in ptc-3(RNAi) animals. Nile Red staining. Scale bars $20 \mu \mathrm{m}$. (K) Quantification of data shown in (J).

Figure 7. Model of how PTC-3 and the loss thereof affects ER structure and LD formation. PTC-3 controls intracellular cholesterol levels directly by promoting its efflux. In the absence of PTC-3, cells accumulate cholesterol, which in turn directly influence membrane properties. In addition, cholesterol directly or indirectly affects NHRs, which subsequently leads to a reduction of acyl chain length and desaturation. This second effect enhances the changes in membrane properties and leads to changes in ER morphology and LD formation.

\section{Supplementary data}

Figure S1. PTC-3 expression pattern. (A) Antibodies against PTC-3 are specific. Immunoblot of lysates of mock or ptc-3 (RNAi) treated worms. (B) PTC-3 gut localization was confirmed with a GFP-tagged PTC-3. Arrows point to the apical intestinal membrane. Scale bars $10 \mu \mathrm{m}$ (C) Mock and ptc-3(RNAi) treated animals were imaged with CARS microscopy. The signal did not overlap with the autofluorescence of LROs, validating the proper spectral separation of the filters, and that we can detect specifically lipid droplets. Scale bars $10 \mu \mathrm{m}$. 
Figure S2. Neither 7-DHC nor DA rescued the ptc-3(RNAi) phenotype. Quantification of number of ptc-3(RNAi) treated worms that reached adulthood when fed from L1 larva under standard cholesterol conditions $(5 \mathrm{mg} / \mathrm{l})$, without cholesterol or if 7-DHC or DA was added for 3 days. Addition of 7-DHC or DA did not alleviate the ptc-3(RNAi) arrested phenotype over the no cholesterol control. Error bars are SEM.

Figure S3. ER morphology appears to be altered upon ptc-3(RNAi). Light microscopy images of intestinally expressed TRAM-GFP. Images suggest morphological alterations in the ER in ptc-3(RNAi) animals. Scale bar $5 \mu \mathrm{m}$.

Figure S4. FIB-SEM analysis of intestinal cell reveal sheet-like ER in ptc-3(RNAi) animals. (A) Workflow of FIB-SEM analysis. After TEM analysis a region of interest was chosen, SEM images were acquire followed by milling steps. A SEM Z-stack was generated, and identification of the ER was done by iLastik training. (B) Representative images showing automatic ER identification in green by iLastik after machine learning training sessions. Scale bars $200 \mathrm{~nm}$.

Figure S5. PTC-3 does not genetically interact with NHR-8 and does not affect SBP-1 localization. (A) $n h r-8(h d 117)$ animals were treated with ptc-3(RNAi) and survival was compared with $n h r-8(h d 117)$ NHR-8::GFP expressing animals. (B) NHR8 over-expressing animals were treated with ptc-3(RNAi) and survival was scored. (C) SBP-1::GFP localization was determined under different RNAi and cholesterol conditions.

Figure S6. Golgi structure is not dramatically affected ptc-3(RNAi) animals. TEM images showing Golgi structure in Mock and ptc-3(RNAi) treated animals. No mayor disruption of the Golgi apparatus was observed. ER: Endoplasmic reticulum, M: mitochondria, G: Golgi

\section{Movies:}

Movie S1. Mock ER-3D reconstruction. In a FIB-SEM Z-stack ER was identified by iLastik training. ER structures showed a reticulated morphology. Scale bar $0.4 \mu \mathrm{m}$ 

available under aCC-BY-ND 4.0 International license.

Movie S2. ptc-3(RNAi) ER-3D reconstruction. In a FIB-SEM Z-stack ER was identified by iLastik training. Upon ptc-3(RNAi) ER-sheets were identified. Scale bar $0.4 \mu \mathrm{m}$. 